\title{
Data report: revised Pleistocene age model based on a modified nannofossil bioevent and additional measurements of benthic foraminiferal oxygen and stable carbon isotope ratios from Hole U1352B, offshore Canterbury Basin, New Zealand'
}

\author{
Koichi Hoyanagi, ${ }^{2}$ Yuichiro Tanaka, ${ }^{3}$ Tokinori Takeuchi, ${ }^{2}$ Minoru Ikehara, ${ }^{4}$ Masayuki Utsunomiya, ${ }^{3}$ \\ Peter Blum, ${ }^{5}$ Cecilia M. McHugh, ${ }^{6}$ and Craig S. Fulthorpe ${ }^{7}$
}

\section{Chapter contents}

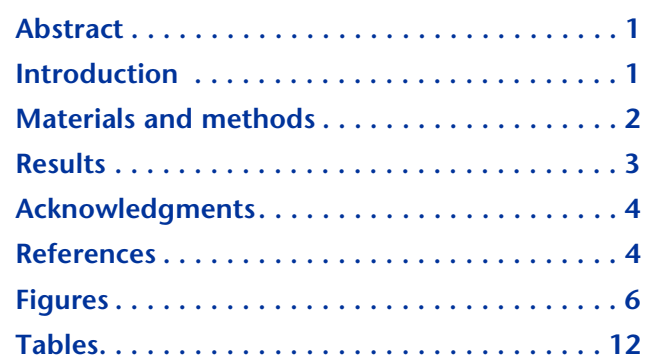

'Hoyanagi, K., Tanaka, Y., Takeuchi, T., Ikehara, M., Utsunomiya, M., Blum, P., McHugh, C.M., and Fulthorpe, C.S., 2018. Data report: revised Pleistocene age model based on a modified nannofossil bioevent and additional measurements of benthic foraminiferal oxygen and stable carbon isotope ratios from Hole U1352B, offshore Canterbury Basin, New Zealand. In Fulthorpe, C.S., Hoyanagi, K., Blum, P., and the Expedition 317 Scientists, Proceedings of the Integrated Ocean Drilling Program, 317: Tokyo (Integrated Ocean Drilling Program Management International, Inc.). doi:10.2204/iodp.proc.317.210.2018

2Department of Geology, Institute of Science, Shinshu University, 3-1-1 Asahi, Matsumoto 3908621, Japan. Correspondence author: hoya101@shinshu-u.ac.jp

${ }^{3}$ Geological Survey of Japan, AIST, 1-1-1 Higashi, Tsukuba 305-8567, Japan.

${ }^{4}$ Center for Advanced Marine Core Research, Kochi University, Nankoku 783-8502, Japan.

${ }^{5}$ International Ocean Discovery Program, Texas A\&M University, College Station, TX 77845, USA. ${ }^{6}$ School of Earth and Environmental Sciences, Queens College, CUNY, 65-30 Kissena Boulevard, Flushing NY 11367, USA.

${ }^{7}$ University of Texas Institute for Geophysics, John A. and Katherine G. Jackson School of Geosciences, J.J. Pickle Research Campus, Building 196 (ROC), 10100 Burnet Road (R2200), Austin TX 787584445, USA.

\section{Abstract}

Reexamination of a nannofossil datum, the highest occurrence of Pseudoemiliania lacunosa (Zone NN20/NN19; 0.44 Ma), has led to a revised datum depth in Integrated Ocean Drilling Program Expedition 317 Hole U1352B. Furthermore, comparison between isotopic ratios of benthic foraminifers Nanionella flemingi and Uvigerina perigrina indicate that oxygen and carbon isotopic ratios for both species, when they occur in the same horizon, are almost identical. Therefore, we can correlate the oxygen isotopic variations of $N$. flemingi with the LR04 stack. We have conducted additional measurements of oxygen and stable carbon isotope ratios of $N$. flemingi, which in conjunction with our revised nannofossil datum allows a new correlation with the LR04 stack and results in revision of our original age model.

\section{Introduction}

Hoyanagi et al. (2014) reported oxygen and stable carbon isotope ratios of the benthic foraminifer Nanionella flemingi in cores from Hole U1352B, which was drilled on the upper slope offshore Canterbury Basin (New Zealand) during Integrated Ocean Drilling Program (IODP) Expedition 317. The oxygen isotope record was correlated with the LR04 stack (Lisiecki and Raymo, 2005) with the support of shipboard bioevent ages (see the "Site U1352" chapter [Expedition 317 Scientists, 2011b]) to generate an age model for the upper $500 \mathrm{~m}$ of Hole U1352B.

Although the nannofossil bioevent highest occurrence (HO) of Pseudoemiliania lacunosa (0.44 Ma) was used for the age model in Hoyanagi et al. (2014), the following problems have since been identified:

- Pseudoemiliania ovata and Pseudoemiliania pacifica were found above the HO of $P$. lacunosa datum (see the "Site U1352" chapter [Expedition 317 Scientists, 2011b]).

- The benthic foraminiferal bioevent, $\mathrm{HO}$ of Bolivinita pliozea (0.6 Ma), was placed above the HO of P. lacunosa (0.44 Ma) in Hole U1352B (i.e., the older age datum was placed above the 
younger age datum). Such a depth reversal for these two bioevent datums was also recognized in Holes U1351B and U1354B.

- The HO of P. lacunosa defines the nannofossil Zone NN20/NN19 boundary and coincides with marine isotope Stage (MIS) 12. However, Hoyanagi et al. (2014) did not find a significant positive oxygen isotopic peak, which would be expected at MIS 12, near the core position originally chosen for the $\mathrm{HO}$ of P. lacunosa during Expedition 317 (i.e., between 155.99 and $164.18 \mathrm{~m}$; all depths are core depth below seafloor, Method A [CSF-A]) (see the "Site U1352" chapter [Expedition 317 Scientists, 2011b]). Hoyanagi et al. (2014) measured oxygen and carbon isotopes every $\sim 1.5 \mathrm{~m}$ in this interval, resulting in samples $<5500 \mathrm{y}$ apart, assuming an average sedimentation rate of $28 \mathrm{~cm} /$ 1000 y (see below). Therefore, we should be able to recognize $\delta^{18} \mathrm{O}$ and $\delta^{13} \mathrm{C}$ fluctuations with cycle periods exceeding $11,000 \mathrm{y}$, which is sufficient to resolve MISs.

Sea level is considered to have risen $135 \mathrm{~m}$ from glacial MIS 12 to interglacial MIS 11 (Miller et al., 2005), so the absence of a positive oxygen isotopic ratio peak at the Zone NN20/NN19 boundary in the Hoyanagi et al. (2014) age model is troubling. Therefore, we have reexamined the occurrence of $P$. lacunosa and have made additional isotopic measurements of benthic foraminifer $N$. flemingi in Hole U1352B cores. These new data indicate that the shipboard depth of the HO of $P$. lacunosa should be revised for the following reasons.

Genus Pseudoemiliania was divided into two species, P. lacunosa and P. ovata, by Young (1998). Specimens of $P$. ovata larger than $6.5 \mu \mathrm{m}$ were further defined as $P$. pacifica by de Kaenel et al. (1999). Usually, the HO of $P$. lacunosa is defined by the last occurrences of the genus Pseudoemiliania (i.e., any of these species), and the $\mathrm{HO}$ of $P$. lacunosa occurs in almost same horizon as the $\mathrm{HO}$ of $P$. ovata (Expedition 315 Scientists, 2009). Expedition 317 Scientists placed the HO of $P$. lacunosa at the horizon between 155.99 and 164.18 $\mathrm{m}$. However, samples at $141.14 \mathrm{~m}$ yield $P$. ovata and $P$. pacifica in the cores from Hole U1352B (see the "Site U1352" chapter [Expedition 317 Scientists, $2011 \mathrm{~b}])$; the HO of P. lacunosa should therefore be no deeper than $141.14 \mathrm{~m}$.

Reexamination of the depth of the HO of $P$. lacunosa in Hole U1352B and additional measurements of benthic foraminiferal oxygen and stable carbon isotope ratios presented in this report lead to a revised correlation with the LR04 stack and a new age model for Hole U1352B. Furthermore, Hoyanagi et al. (2014) did not evaluate the vital effect of $N$. flemingi. We therefore have conducted new measurements of oxygen and stable carbon isotopic ratios of the benthic foraminifer Uvigerina perigrina, which occurs rarely in the cores, to ensure that correlation of isotopic measurements made on $N$. flemingi with the LR04 stack is justified.

Our new results suggest that the original age model (Hoyanagi et al., 2014) should be modified in the intervals between 115 and $215 \mathrm{~m}$ and between 255 and $305 \mathrm{~m}$. The former age model is unchanged outside these intervals. All data presented in Hoyanagi et al. (2014) are duplicated in this report.

\section{Materials and methods}

We picked 36 samples for microscopic analysis of calcareous nannofossils every 0.83 to $4.52 \mathrm{~m}$, with an average spacing of $1.5 \mathrm{~m}$ or one sample per section, from 180.4 to $122.37 \mathrm{~m}$ in Hole U1352B (Table T1) to determine the $\mathrm{HO}$ of $P$. lacunosa (Zone NN20/ NN19).

Smear slides were examined under a polarized microscope at 1250× magnification. Three hundred calcareous nannofossils were identified to species level in each slide. The total abundance of calcareous nannofossils was defined as follows:

$\mathrm{A}=$ abundant; usually $>10$ specimens observed per field of view (FOV).

$\mathrm{C}=$ common; $1-10$ specimens per FOV.

$\mathrm{F}=$ few; 1 specimen per FOV.

$\mathrm{R}=$ rare; 1 specimen per $1-10$ FOVs.

$\mathrm{VR}=$ very rare; $<1$ specimen per 10 FOVs.

$\mathrm{B}=$ barren; no nannofossils.

Calcareous nannofossil preservation was recorded using the following criteria (see the "Methods" chapter [Expedition 317 Scientists, 2011a]):

$\mathrm{M}=$ moderate; some etching and/or recrystallization; primary morphological characteristics partially altered; most specimens identifiable at the species level.

$\mathrm{P}=$ poor; specimens severely etched or overgrown; primary morphological characteristics largely destroyed; fragmentation evident; most specimens not identifiable at the species and/or generic level.

Analyses of oxygen and stable carbon isotope ratios of benthic foraminifer $N$. flemingi were carried out on 23 samples from 62-68 m, 8 samples from 95-100 m, 41 samples from 123-151 m, 20 samples from 197$202 \mathrm{~m}, 21$ samples from 247-253 m, 22 samples from $426-432 \mathrm{~m}$, and 42 samples from $446-456 \mathrm{~m}$. A total of 177 samples were measured. The spacing between our new measurements ranges from a mini- 
mum of $0.2 \mathrm{~m}$ to a maximum of $1.45 \mathrm{~m}$ and averages $0.5 \mathrm{~m}$ for $123-151 \mathrm{~m}$, where the focus was on the $\mathrm{HO}$ of $P$. lacunosa and MIS 12 (Table T2). This $0.5 \mathrm{~m}$ sample interval represents $\sim 3300 \mathrm{y}$.

Several $U$. perigrina tests were picked from each of 88 samples from Hole U1352B between 156.4 and 501 $\mathrm{m}$. We measured their oxygen and carbon isotope ratios and compared the ratios with those of $N$. flemingi from the same horizons (Table T3).

Oxygen and stable carbon isotopic analyses were carried out at the Kochi Core Center using an IsoPrime mass spectrometer. We used the same sample preparation and analytical procedures as Hoyanagi et al. (2014). Analytical precision is better than $0.08 \%$ for $\delta^{18} \mathrm{O}$ and $0.05 \%$ for $\delta^{13} \mathrm{C}$.

\section{Results}

\section{Nannofossil datum reexamination}

Results of microscopic observation of nannofossil occurrences are shown in Table T1. P. lacunosa is found from 128.35 to $180.06 \mathrm{~m}$. P. ovata is found from 130.34 to $159.47 \mathrm{~m}$. P. pacifica was observed in two samples at 157.86 and $175.07 \mathrm{~m}$ (Table T1; Fig. F1). Therefore, we confirmed that the occurrences of $P$. lacunosa and/or $P$. ovata are as shallow as $131.98 \mathrm{~m}$ (Table T1). The isolated occurrence of $P$. lacunosa var. lacunosa at $128.35 \mathrm{~m}$ is considered to be reworked. Therefore, we propose that the HO of $P$. lacunosa (Zone NN20/NN19; $0.44 \mathrm{Ma}$ ) in Hole U1352B be placed between 129.77 and $130.34 \mathrm{~m}$ (Table T4).

\section{Impact of new isotopic measurements}

The measured interval between 123 and $151 \mathrm{~m}$ includes our revised depth of the HO of P. lacunosa $(131.98 \mathrm{~m})$. Oxygen isotope ratios were relatively high throughout this interval in Hoyanagi et al. (2014). In contrast, our new measurements resolve two additional positive peaks and one new negative excursion within this interval (Fig. F2). However, the new measurements reveal no new peaks outside this interval.

Although the average sedimentation rate in the upper $500 \mathrm{~m}$ of Hole U1352B is $28 \mathrm{~cm} / \mathrm{ky}$, a lower rate $(17 \mathrm{~cm} / \mathrm{ky})$ is estimated for the interval between the HO of $P$. lacunosa and the lowest occurrence (LO) of Emiliania huxleyi (121.1 m). Hoyanagi et al. (2014) measured isotopic ratios at $1.5 \mathrm{~m}$ intervals. This interval represents $\sim 5400 \mathrm{y}$ with a sedimentation rate of $28 \mathrm{~cm} / \mathrm{ky}$ but $\sim 8800 \mathrm{y}$ with a rate of $17 \mathrm{~cm} / \mathrm{ky}$. Hoyanagi et al. (2014) could therefore have missed several oxygen isotopic ratio peaks in the interval where the sedimentation rate is low. The addition of
41 new measurements in the key 123-151 m interval to the original 58 measurements of Hoyanagi et al. (2014) increases measurement resolution to $\sim 3000 \mathrm{y}$ with a $17 \mathrm{~cm} / \mathrm{ky}$ sedimentation rate and led to resolution of the previously undetected peaks.

Comparison of oxygen and stable carbon isotopic ratios for $N$. flemingi and $U$. perigrina is shown in Figure F2. The oxygen isotope ratios of $N$. flemingi have a strong correlation with those of $U$. perigrina (Fig. F3). The relationship between oxygen isotopic ratios of $N$. flemingi $(Y)$ and $U$. perigrina $(X)$ is expressed by the equation $Y=X+0.27$. Therefore, oxygen isotope ratios of $N$. flemingi and $U$. perigrina can essentially be used interchangeably. The stable carbon isotopic ratios of the two species also show a positive correlation (Fig. F4). Because U. perigrina is considered to have the same oxygen and stable carbon isotopic ratios as the surrounding seawater (Shackleton and Hall, 1984), N. flemingi must also have the same or very similar oxygen and carbon isotopic ratios as those of ambient seawater and can therefore be correlated with the LR04 stack, which is based on $U$. perigrina.

\section{Correlation with the LR04 stack and revised age model}

The HO of P. lacunosa marks the top of Zone NN19 (Martini, 1971) and the top of Subzone CN14a (Okada and Bukry, 1980). Previous studies have demonstrated the worldwide synchroneity of this datum, which corresponds to MIS 12 (e.g., Thierstein et al., 1977; Wei, 1993; Raffi et al., 2006). Our new oxygen isotope measurements show new positive peaks at $\sim 130 \mathrm{~m}\left(\delta^{18} \mathrm{O}=4.023 \%\right.$ at $\left.129.90 \mathrm{~m}\right)$ and $\sim 137 \mathrm{~m}\left(\delta^{18} \mathrm{O}=3.825 \%\right.$ at $\left.137.08 \mathrm{~m}\right)$ and a new negative peak at $\sim 135 \mathrm{~m}\left(\delta^{18} \mathrm{O}=3.059 \%\right.$ ot $\left.134.84 \mathrm{~m}\right)$ (Fig. F2; Table T2). Using our revised HO of $P$. lacunosa (0.44 Ma) at $131.98 \mathrm{~m}$ (Table T4), we correlate these new positive peaks with MIS 12 and 14 and the negative peak with MIS 13. Our new correlations show a clear positive peak in $\delta^{18} \mathrm{O}$ at the $\mathrm{HO}$ of $P$. lacunosa that is concordant with the timescale of Lourens et al. (2004).

Expedition 317 Scientists suggested that a hiatus from 1.8 to 2.7 Ma lies somewhere between 491.74 and $525.34 \mathrm{~m}$. Therefore, we use the eight Pleistocene nannofossil datum levels shallower than 491.74 m (Table T4; see the "Site U1352" chapter [Expedition 317 Scientists, 2011b]) to correlate the $\delta^{18} \mathrm{O}$ record (Fig. F5A) with the global benthic foraminiferal $\delta^{18} \mathrm{O}$ LR04 stack of Lisiecki and Raymo (2005) (Fig. F5B) and to plot the Expedition $317 \delta^{18} \mathrm{O}$ record versus age from $1.8 \mathrm{Ma}$ to present. 
We made revised correlations relative to Hoyanagi et al. (2014) for the intervals between MIS 11 and 17 and between MIS 25 and 29 (Fig. F5C). N. flemingi tests were present in the interval between 62 and 66 $\mathrm{m}$ but were not present between 10.7 and $62 \mathrm{~m}$. Therefore, we cannot correlate our record to MIS 4 of the LR04 stack. Other correlations of Hoyanagi et al. (2014) between the Hole U1352B oxygen isotope record and the LR04 stack were left unchanged (Fig. F5).

Figure $\mathrm{F} 6$ shows the sedimentation rates (age model) for Hole U1352B. The ages within the intervals between 115 and $215 \mathrm{~m}$ and between 255 and $305 \mathrm{~m}$ are shifted relative to the ages in Hoyanagi et al. (2014). Maximum age differences between the two models is $\sim 0.2 \mathrm{my}$ for the same depth. The new age model (Fig. F6) indicates that the average sedimentation rate in the upper $\sim 500 \mathrm{~m}$ of Hole U1352B is $\sim 28$ $\mathrm{cm} / 1000 \mathrm{y}$. However, sedimentation rates fluctuate; the maximum rate is $\sim 76 \mathrm{~cm} / 1000 \mathrm{y}$ between 0.8 and $0.7 \mathrm{Ma}$ and the minimum rate is $\sim 3 \mathrm{~cm} / 1000 \mathrm{y}$ between 0.9 and $0.8 \mathrm{Ma}$.

\section{Acknowledgments}

This research used samples and data provided by the Integrated Ocean Drilling Program (IODP). We acknowledge the IODP Expedition 317 shipboard scientists, technical staff, and the captain and crew of the R/V JOIDES Resolution. We would like to thank Clara T. Bolton and an anonymous reviewer. This article was greatly improved by their detailed review and valuable suggestions. Oxygen and stable carbon isotopic measurements were performed under the cooperative research program of the Center for Advanced Marine Core Research (CMCR), Kochi University (Numbers 13A034, 13B028, 14A031, 14B029, 15A039, and 15B034). Funding for this research was provided by Japan Society for the Promotion of Science (JSPS Grants-in Aid for Scientific Research Number 23340154).

\section{References}

de Kaenel, E., Siesser, W.G., and Murat, A., 1999. Pleistocene calcareous nannofossil biostratigraphy and the western Mediterranean sapropels, Sites 974 to 977 and 979. In Zahn, R., Comas, M.C., and Klaus, A. (Eds.), Proceedings of the Ocean Drilling Program, Scientific Results, 161: College Station, TX (Ocean Drilling Program), 159-183. https:// doi.org/10.2973/odp.proc.sr.161.250.1999

Expedition 315 Scientists, 2009. Expedition 315 Site C0002. In Kinoshita, M., Tobin, H., Ashi, J., Kimura, G., Lallemant, S., Screaton, E.J., Curewitz, D., Masago, H., Moe, K.T., and the Expedition 314/315/316 Scientists, Proceedings of the Integrated Ocean Drilling Program, 314/
315/316: Washington, DC (Integrated Ocean Drilling Program Management International, Inc.). https:// doi.org/10.2204/iodp.proc.314315316.124.2009

Expedition 317 Scientists, 2011a. Methods. In Fulthorpe, C.S., Hoyanagi, K., Blum, P., and the Expedition 317 Scientists, Proceedings of the Integrated Ocean Drilling Program, 317: Tokyo (Integrated Ocean Drilling Program Management International, Inc.). https://doi.org/ 10.2204/iodp.proc.317.102.2011

Expedition 317 Scientists, 2011b. Site U1352. In Fulthorpe, C.S., Hoyanagi, K., Blum, P., and the Expedition 317 Scientists, Proceedings of the Integrated Ocean Drilling Program, 317: Tokyo (Integrated Ocean Drilling Program Management International, Inc.). https://doi.org/ 10.2204/iodp.proc.317.104.2011

Hoyanagi, K., Kawagata, S., Koto, S., Kamihashi, T., and Ikehara, M., 2014. Data report: Pleistocene benthic foraminiferal oxygen and stable carbon isotopes and their application for age models, Hole U1352, offshore New Zealand. In Fulthorpe, C.S., Hoyanagi, K., Blum, P., and the Expedition 317 Scientists, Proceedings of the Integrated Ocean Drilling Program, 317: Tokyo (Integrated Ocean Drilling Program Management International, Inc.). https://doi.org/10.2204/iodp.proc.317.208.2014

Kameo, K., and Bralower, T.J., 2000. Neogene calcareous nannofossil biostratigraphy of Sites 998, 999, and 1000, Caribbean Sea. In Leckie, R.M., Sigurdsson, H., Acton, G.D., and Draper, G. (Eds.), Proceedings of the Ocean Drilling Program, Scientific Results, 165: College Station, TX (Ocean Drilling Program), 3-17. https://doi.org/ 10.2973/odp.proc.sr.165.012.2000

Lisiecki, L.E., and Raymo, M.E., 2005. A Pliocene-Pleistocene stack of 57 globally distributed benthic $\delta^{18} \mathrm{O}$ records. Paleoceanography, 20(1):PA1003. https:// doi.org/10.1029/2004PA001071

Lourens, L., Hilgen, F., Shackleton, N.J., Laskar, J., and Wilson, D., 2004. The Neogene period. In Gradstein, F.M., Ogg, J.G., and Smith, A. (Eds.), A Geologic Time Scale 2004: Cambridge, UK (Cambridge Univ. Press), 409440. https://doi.org/10.1017/ CBO9780511536045.022

Martini, E., 1971. Standard Tertiary and Quaternary calcareous nannoplankton zonation. In Farinacci, A. (Ed.), Proceeding of the Second Planktonic Conference Roma 1970: Rome (Edizioni Tecnoscienza), 2:739-785.

Miller, K.G., Kominz, M.A., Browning, J.V., Wright, J.D., Mountain, G.S., Katz, M.E., Sugarman, P.J., Cramer, B.S., Christie-Blick, N., and Pekar, S.F., 2005. The Phanerozoic record of global sea-level change. Science, 310(5752):1293-1298. https://doi.org/10.1126/science. 1116412

Okada, H., and Bukry, D., 1980. Supplementary modification and introduction of code numbers to the low-latitude coccolith biostratigraphic zonation (Bukry, 1973; 1975). Marine Micropaleontology, 5:321-325. https:// doi.org/10.1016/0377-8398(80)90016-X

Paillard, D., Labeyrie, L., and Yiou, P., 1996. Macintosh program performs time-series analysis. Eos, Transactions of the American Geophysical Union, 77(39):379. https:// doi.org/10.1029/96EO00259 
Raffi, I., Backman, J., Fornaciari, E., Pälike, H., Rio, D., Lourens, L., and Hilgen, F., 2006. A review of calcareous nannofossil astrobiochronology encompassing the past 25 million years. Quaternary Science Reviews, 25(2324):3113-3137. https://doi.org/10.1016/j.quascirev.2006.07.007

Shackleton, N.J., and Hall, M.A., 1984. Oxygen and carbon isotope stratigraphy of Deep Sea Drilling Project Hole 552A: Plio-Pleistocene glacial history. In Roberts, D.G., Schnitker, D., et al., Initial Reports of the Deep Sea Drilling Project, 81: Washington, DC (U.S. Government Printing Office), 599-609. https://doi.org/10.2973/ dsdp.proc.81.116.1984

Thierstein, H.R., Geitzenauer, K.R., Molfino, B., and Shackleton, N.J., 1977. Global synchroneity of late Quater- nary coccolith datum levels validation by oxygen isotopes. Geology, 5(7):400-404. https://doi.org/ 10.1130/0091-7613(1977)5<400:GSOLQC>2.0.CO;2

Young, J.R., 1998. Neogene. In Bown, P.R. (Ed.), Calcareous Nannofossil Biostratigraphy: Dordrecht, The Netherlands (Kluwer Academic Publishing), 225-265.

Wei, W., 1993. Calibration of upper Pliocene-lower Pleistocene nannofossil events with oxygen isotope stratigraphy. Paleoceanography, 8(1):85-99. https://doi.org/ 10.1029/92PA02504

Initial receipt: 27 March 2016

Acceptance: 22 June 2018

Publication: 7 September 2018

MS 317-210 
Figure F1. Selected calcareous nannofossil species, Hole U1352B. Scale bars $=3 \mu \mathrm{m}$. A, B. Pseoudemilianica lacunosa (Kamptner) Gartner (139.25 m CSF-A). C. Pseoudemilianica ovata (Bukry) Young (157.86 m CSF-A). D. Gephyrocapsa oceanica Kamptner (128.35 m CSF-A).
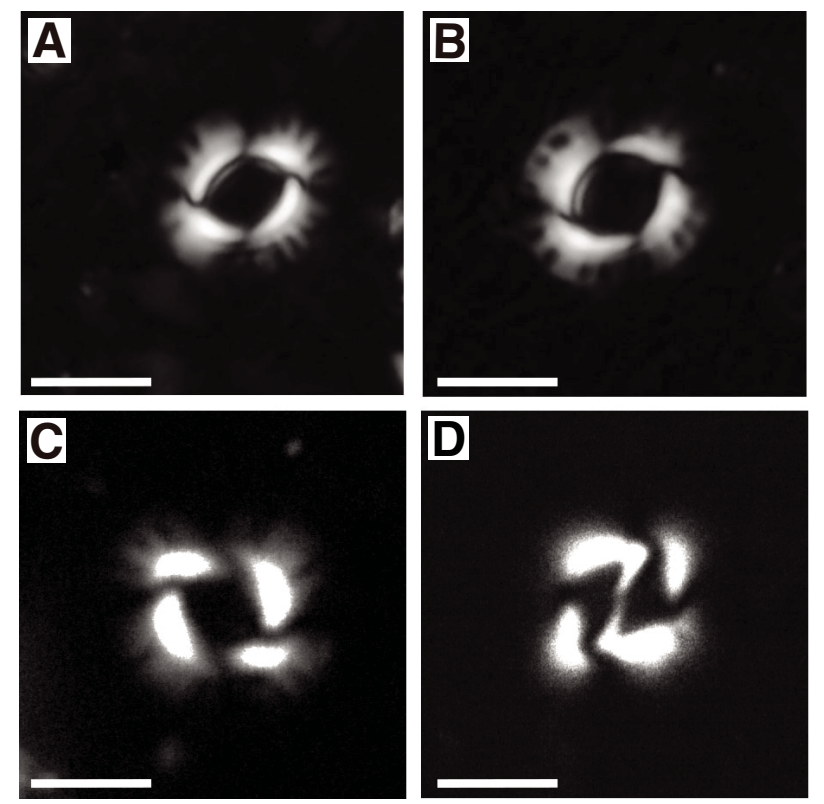
Figure F2. Oxygen and carbon isotope ratios of foraminifer Nonionella flemingi tests from 520 samples (solid lines), including data reported in Hoyanagi et al. (2014), and those of Uvigerina perigrina tests from 87 samples (dashed lines), Hole U1352B. Solid circles $=$ measured values. Orange $=\delta^{13} \mathrm{C}$, blue $=\delta^{18} \mathrm{O} . \mathrm{VPDB}=$ Vienna Peedee belemnite.

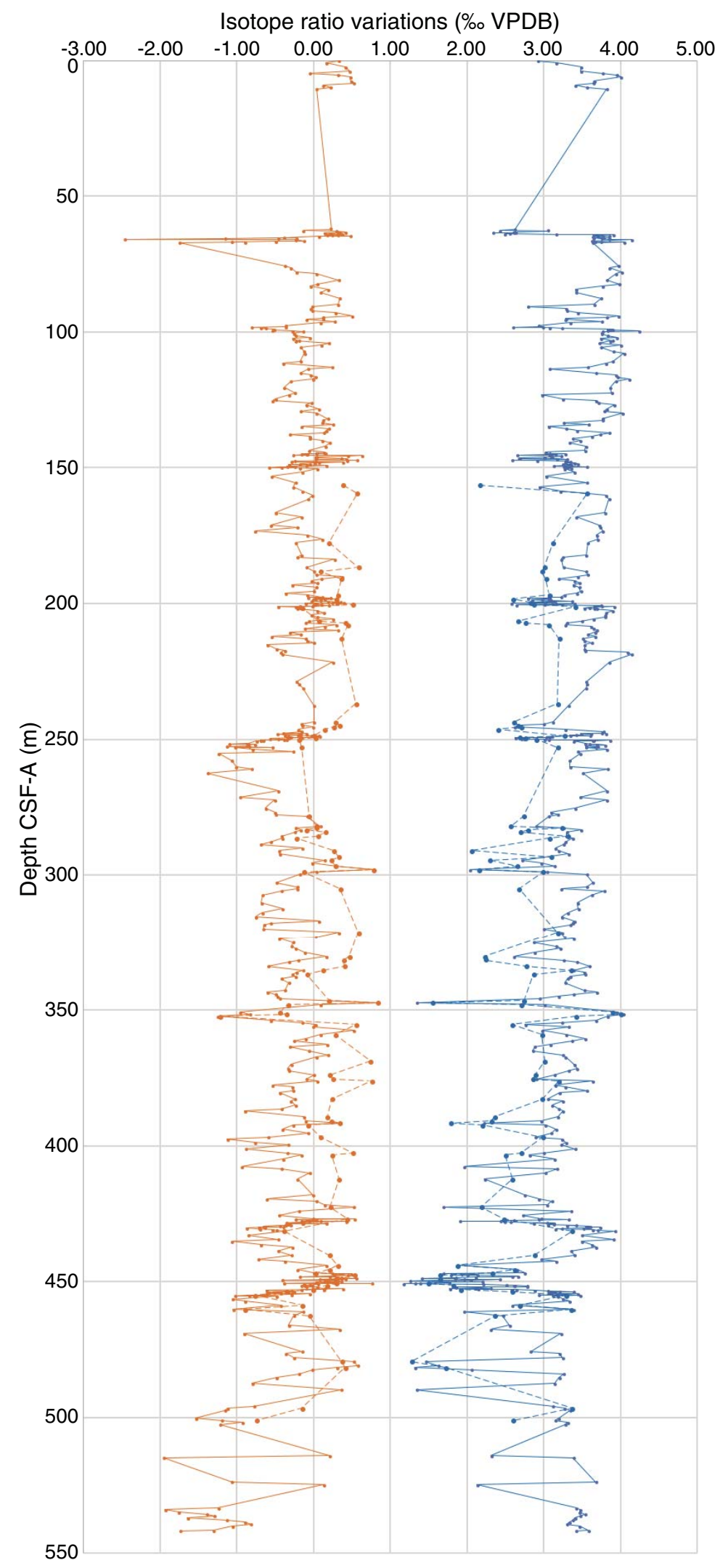


Figure F3. Comparison between oxygen isotopic measurements made on Nonionella flemingi and Uvigerina perigrina, Hole U1352B. All N. flemingi values, except that at $249.48 \mathrm{~m}$ CSF-A, are from Hoyanagi et al. (2014). $\mathrm{VPDB}=$ Vienna Peedee belemnite.

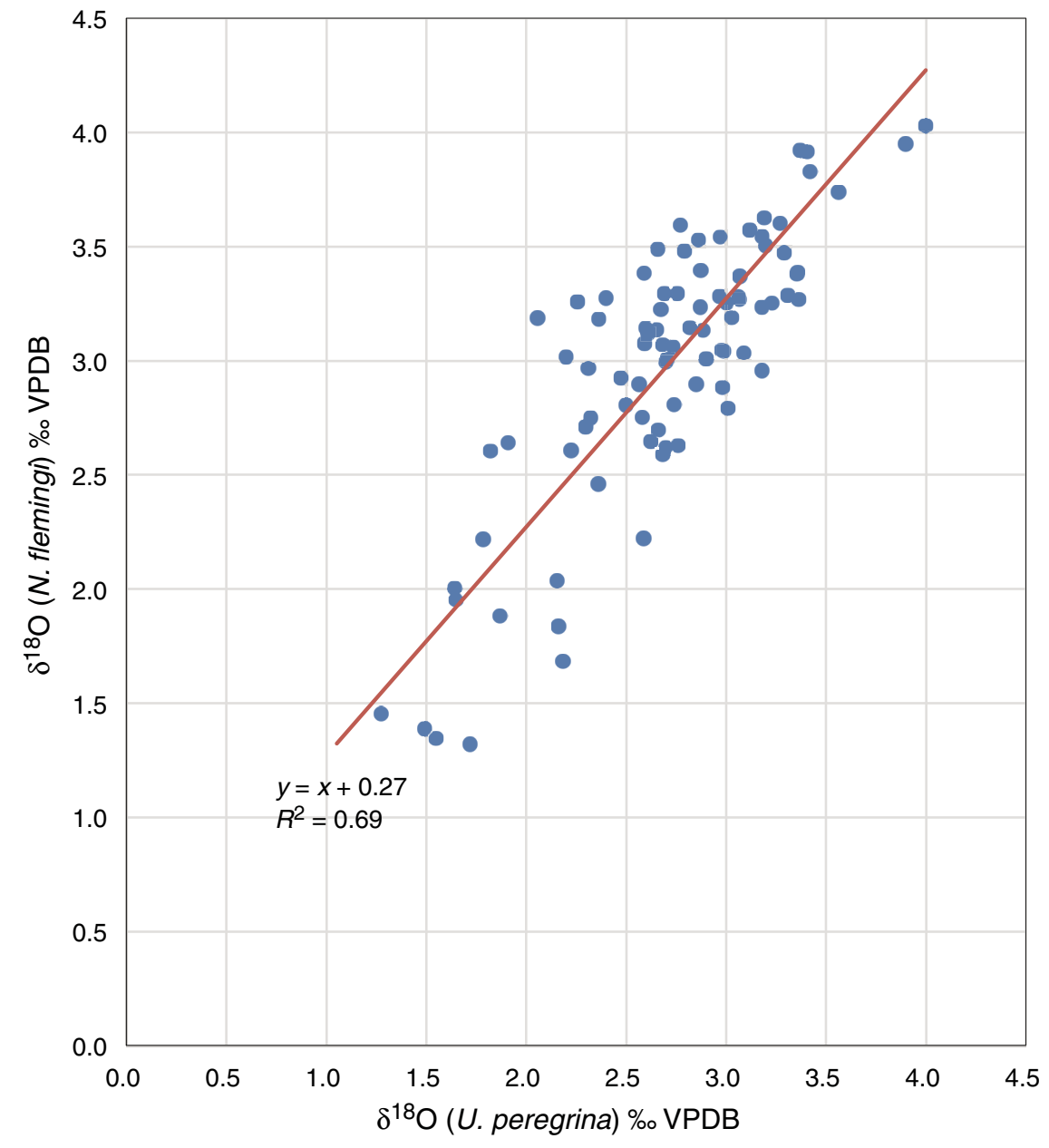


Figure F4. Comparison between carbon isotopic measurements made on Nonionella flemingi and Uvigerina perigrina, Hole U1352B. All N. flemingi values, except that at $249.48 \mathrm{~m}$ CSF-A, are from Hoyanagi et al. (2014). $\mathrm{VPDB}=$ Vienna Peedee belemnite.

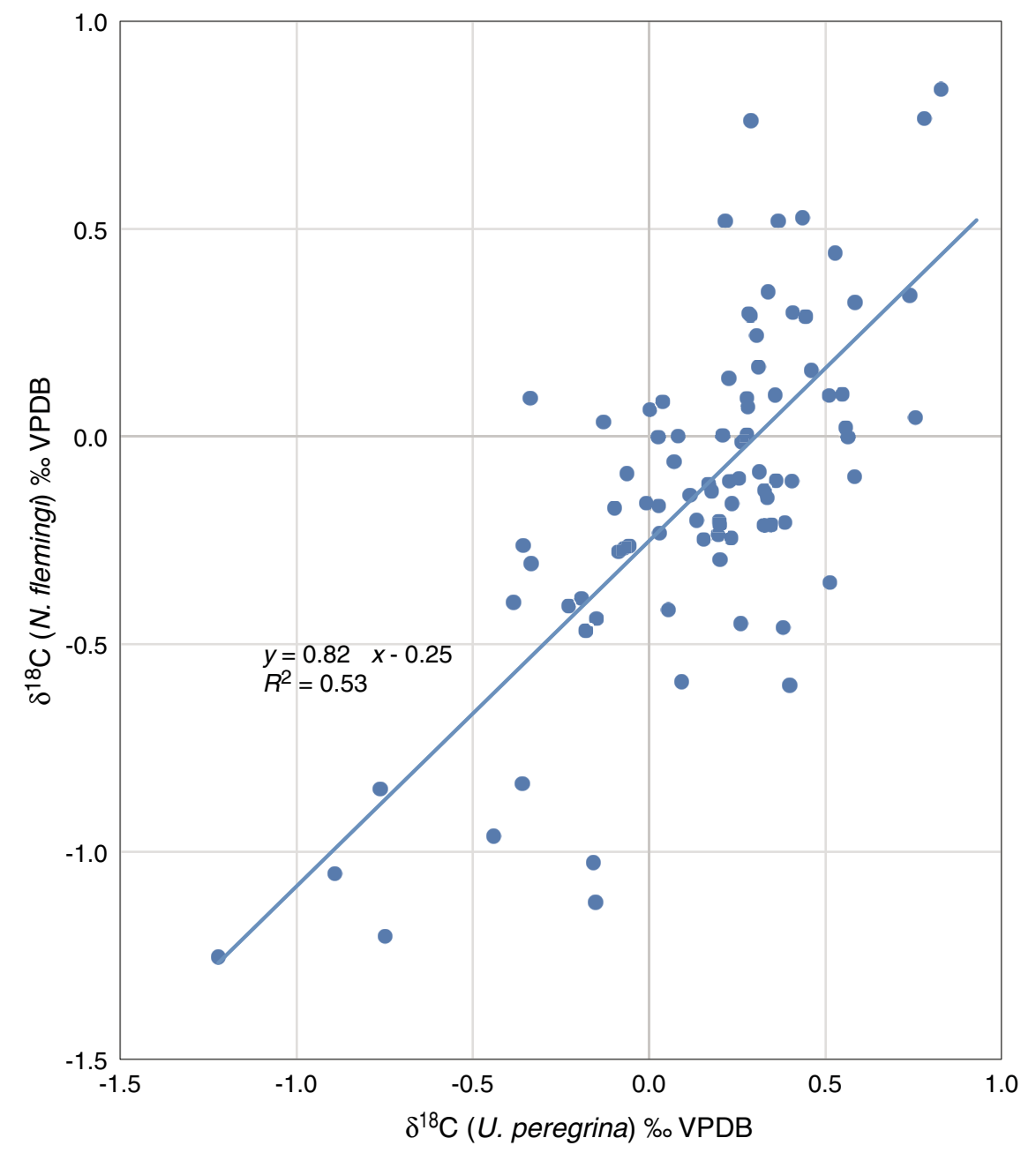


Figure F5. Oxygen isotope variations and LR04 stack (Lisiecki and Raymo, 2005), Site U1352. Numbers adjacent to data curves are marine isotope stages. A. 0-502 m CSF-A. Orange bars and lines = nannofossil datum ages (see Table T4). $\mathrm{LO}=$ lowest occurrence, $\mathrm{HO}=$ highest occurrence, $\mathrm{HCO}=$ highest common occurrence. Numbers in parentheses are ages (Ma). B. LR04 stack. C. Rescaled oxygen isotope variations (AnalySeries 2.0.4.3 software; Paillard et al., 1996) versus age. Red MIS numbers = correlations that are new and differ from those of Hoyanagi et al. (2014).

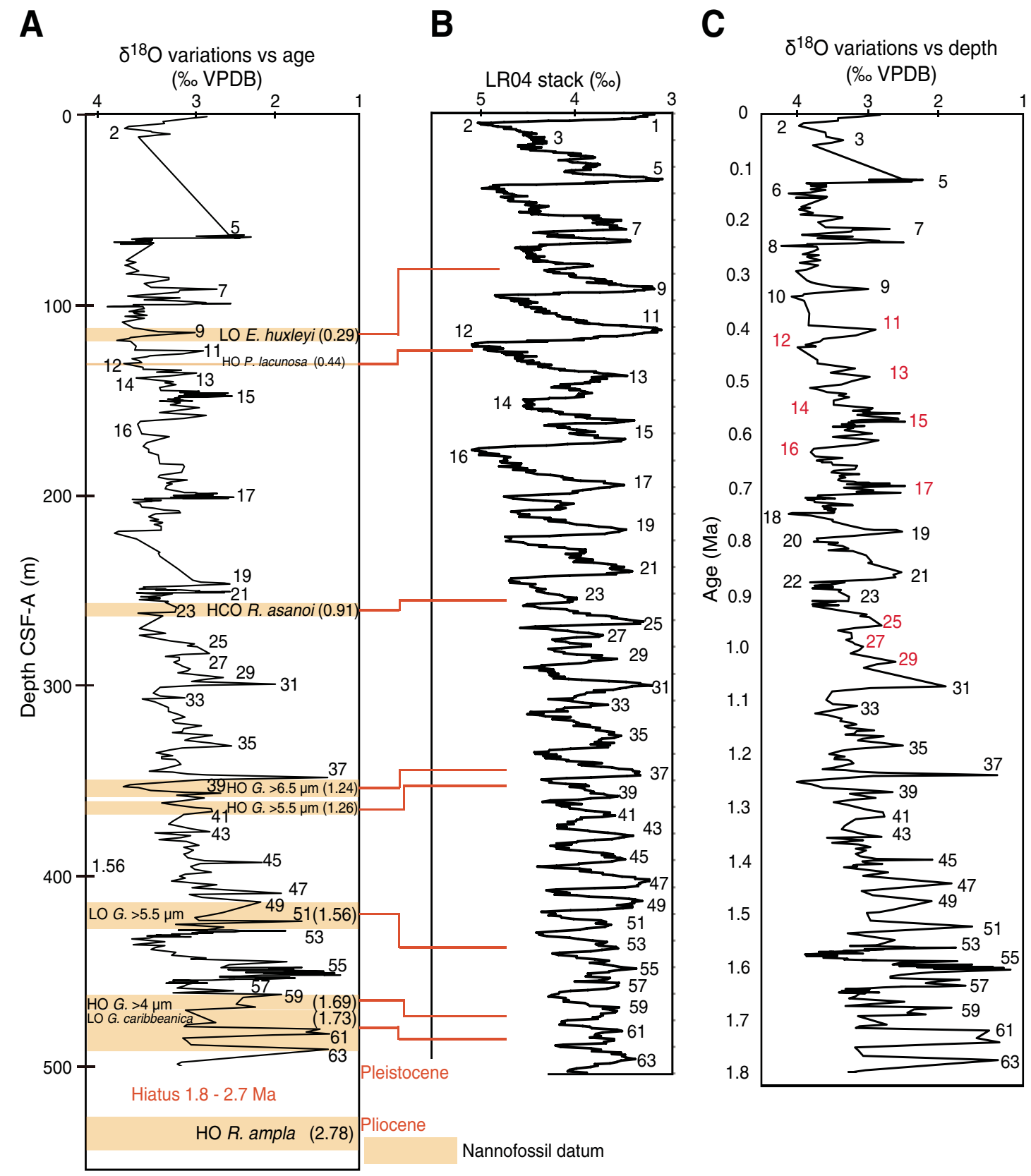


Figure F6. Sedimentation rates, Hole U1352B. Thick line = data produced using AnalySeries 2.0.4.3 software (Paillard et al., 1996), thin line = data from Hoyanagi et al. (2014), dotted line = average sedimentation rate for upper $500 \mathrm{~m}$ CSF-A.

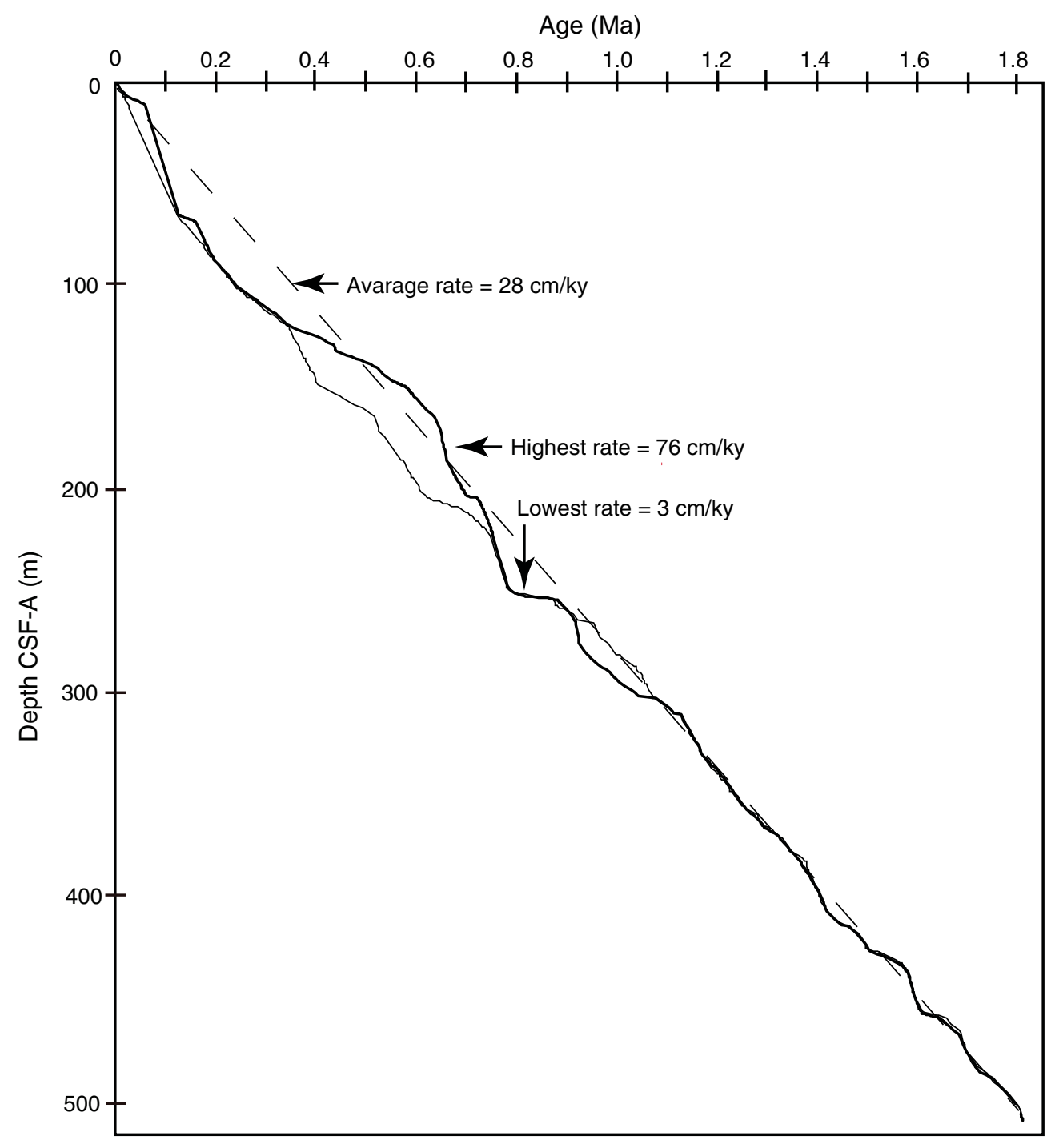


Table T1. Calcareous nannofossils, Hole U1352B.

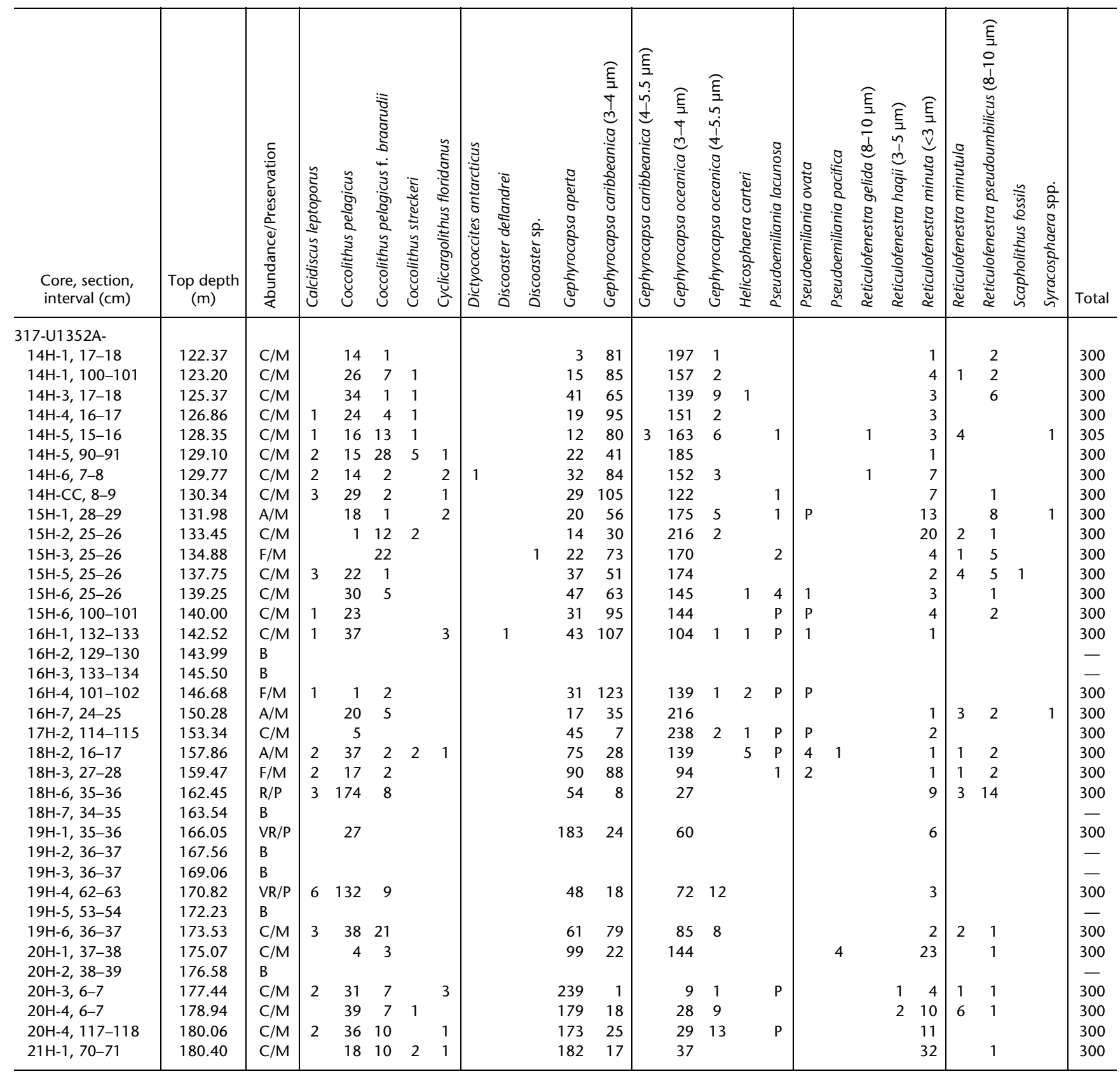

Abundance: $A=$ abundant, $C=$ common, $F=$ few, $R=$ rare, $V R=$ very rare, $P=$ present outside the count, $B=$ barren (no nannofossils). Preservation: $\mathrm{M}=$ moderate, $\mathrm{P}=$ poor. 
Table T2. Oxygen isotopic ratios $\left(\delta^{18} \mathrm{O}\right)$ and stable carbon isotopic ratios $\left(\delta^{13} \mathrm{C}\right)$ of Nanionella flemingi from this study (177 samples) and Hoyanagi et al. (2014) (shaded cells; 341 samples), Hole U1352B. (Continued on next seven pages.)

\begin{tabular}{|c|c|c|c|c|c|}
\hline $\begin{array}{l}\text { Core, section, } \\
\text { interval }(\mathrm{cm})\end{array}$ & $\begin{array}{l}\text { Top depth } \\
\text { CSF-A (m) }\end{array}$ & $\begin{array}{l}\text { Middle depth } \\
\text { CSF-A (m) }\end{array}$ & $\begin{array}{l}\text { Bottom depth } \\
\text { CSF-A (m) }\end{array}$ & $\begin{array}{c}\delta^{13} \mathrm{C} \\
\text { (\%o VPDB) }\end{array}$ & $\begin{array}{c}\delta^{18} \mathrm{O} \\
(\% \circ \text { VPDB) }\end{array}$ \\
\hline \multicolumn{6}{|l|}{ 317-U1352B- } \\
\hline $1 \mathrm{H}-1,19-21$ & 0.10 & 0.20 & 0.30 & 0.320 & 2.918 \\
\hline $1 \mathrm{H}-1,94-96$ & 0.85 & 0.95 & 1.05 & 0.161 & 3.161 \\
\hline $1 \mathrm{H}-2,94-186$ & 2.35 & 2.45 & 2.55 & 0.407 & 3.484 \\
\hline $1 \mathrm{H}-3,94-96$ & 3.85 & 3.95 & 4.05 & 0.469 & 3.480 \\
\hline $1 \mathrm{H}-4,19-21$ & 4.60 & 4.70 & 4.80 & -0.054 & 3.762 \\
\hline $1 \mathrm{H}-4,94-96$ & 5.35 & 5.45 & 5.55 & 0.315 & 3.941 \\
\hline $1 \mathrm{H}-5,19-21$ & 6.10 & 6.20 & 6.30 & 0.472 & 4.005 \\
\hline $1 \mathrm{H}-6,19-21$ & 7.50 & 7.60 & 7.70 & 0.483 & 3.651 \\
\hline $2 \mathrm{H}-1,19-21$ & 8.30 & 8.40 & 8.50 & 0.517 & 3.638 \\
\hline $2 \mathrm{H}-1,94-96$ & 9.05 & 9.15 & 9.25 & 0.122 & 3.408 \\
\hline $2 \mathrm{H}-2,20-22$ & 9.81 & 9.91 & 10.01 & 0.219 & 3.561 \\
\hline $2 \mathrm{H}-2,94-96$ & 10.55 & 10.65 & 10.75 & 0.037 & 3.811 \\
\hline $7 \mathrm{H}-5,46-48$ & 62.16 & 62.17 & 62.18 & 0.234 & 2.621 \\
\hline $7 \mathrm{H}-5,92-94$ & 62.62 & 62.63 & 62.64 & -0.136 & 2.423 \\
\hline $7 \mathrm{H}-5,115-117$ & 62.85 & 62.86 & 62.87 & 0.292 & 3.062 \\
\hline $7 \mathrm{H}-6,5-7$ & 63.25 & 63.26 & 63.27 & 0.408 & 2.603 \\
\hline $7 \mathrm{H}-6,19-21$ & 63.30 & 63.40 & 63.50 & 0.332 & 2.625 \\
\hline $7 \mathrm{H}-6,25-27$ & 63.45 & 63.46 & 63.47 & 0.318 & 2.339 \\
\hline $7 \mathrm{H}-6,45-47$ & 63.65 & 63.66 & 63.67 & 0.14 & 2.551 \\
\hline $7 \mathrm{H}-6,71-73$ & 63.91 & 63.92 & 63.93 & 0.218 & 2.487 \\
\hline $7 \mathrm{H}-6,85-87$ & 64.05 & 64.06 & 64.07 & 0.367 & 3.153 \\
\hline $7 \mathrm{H}-6,94-96$ & 64.05 & 64.15 & 64.25 & 0.233 & 3.751 \\
\hline 7H-6, 104-106 & 64.24 & 64.25 & 64.26 & 0.172 & 3.907 \\
\hline $7 \mathrm{H}-6,123-125$ & 64.43 & 64.44 & 64.45 & 0.316 & 3.638 \\
\hline $7 \mathrm{H}-\mathrm{CC}, 23-25$ & 64.68 & 64.69 & 64.70 & 0.479 & 3.849 \\
\hline 7H-CC, 38-40 & 64.83 & 64.84 & 64.85 & 0.061 & 3.636 \\
\hline $8 \mathrm{H}-1,5-7$ & 65.25 & 65.26 & 65.27 & -0.388 & 3.778 \\
\hline $8 \mathrm{H}-1,19-21$ & 65.30 & 65.40 & 65.50 & -0.229 & 3.847 \\
\hline $8 \mathrm{H}-1,25-27$ & 65.45 & 65.46 & 65.47 & -0.461 & 3.648 \\
\hline $8 \mathrm{H}-1,45-47$ & 65.65 & 65.66 & 65.67 & -1.157 & 3.747 \\
\hline $8 \mathrm{H}-1,68-70$ & 65.88 & 65.89 & 65.90 & -2.461 & 3.846 \\
\hline $8 \mathrm{H}-1,85-87$ & 66.05 & 66.06 & 66.07 & -0.239 & 4.139 \\
\hline $8 \mathrm{H}-1,134-136$ & 66.54 & 66.55 & 66.56 & -0.128 & 3.623 \\
\hline $8 \mathrm{H}-1,145-147$ & 66.65 & 66.66 & 66.67 & -0.5 & 3.662 \\
\hline $8 \mathrm{H}-2,20-22$ & 66.81 & 66.91 & 67.01 & -0.897 & 4.046 \\
\hline $8 \mathrm{H}-2,13-15$ & 66.83 & 66.84 & 66.85 & -1.065 & 3.745 \\
\hline $8 \mathrm{H}-2,35-37$ & 67.05 & 67.06 & 67.07 & -1.748 & 3.634 \\
\hline $9 \mathrm{H}-1,94-96$ & 75.55 & 75.65 & 75.75 & -0.373 & 3.973 \\
\hline $9 \mathrm{H}-2,19-21$ & 76.30 & 76.40 & 76.50 & -0.298 & 3.853 \\
\hline $9 \mathrm{H}-3,19-21$ & 77.80 & 77.90 & 78.00 & -0.228 & 4.007 \\
\hline $9 \mathrm{H}-3,94-96$ & 78.55 & 78.65 & 78.75 & 0.030 & 3.939 \\
\hline $9 \mathrm{H}-5,19-21$ & 80.80 & 80.90 & 81.00 & 0.321 & 3.818 \\
\hline $9 \mathrm{H}-6,18-20$ & 82.29 & 82.39 & 82.49 & 0.044 & 3.980 \\
\hline $9 \mathrm{H}-6,92-94$ & 83.03 & 83.13 & 83.23 & -0.047 & 3.757 \\
\hline $10 \mathrm{H}-1,19-21$ & 84.30 & 84.40 & 84.50 & 0.180 & 3.416 \\
\hline $10 \mathrm{H}-2,95-97$ & 85.32 & 85.42 & 85.52 & 0.091 & 3.421 \\
\hline $10 \mathrm{H}-4,19-21$ & 87.56 & 87.66 & 87.76 & 0.338 & 3.746 \\
\hline $10 \mathrm{H}-5,94-96$ & 89.81 & 89.91 & 90.01 & 0.317 & 3.649 \\
\hline $10 \mathrm{H}-6,19-21$ & 90.56 & 90.66 & 90.76 & -0.021 & 2.788 \\
\hline $10 \mathrm{H}-6,94-96$ & 91.31 & 91.41 & 91.51 & -0.040 & 3.283 \\
\hline $10 \mathrm{H}-7,20-22$ & 92.07 & 92.17 & 92.27 & -0.024 & 3.299 \\
\hline $10 \mathrm{H}-7,94-96$ & 92.81 & 92.91 & 93.01 & 0.279 & 3.434 \\
\hline $11 \mathrm{H}-1,51-53$ & 94.12 & 94.22 & 94.32 & 0.498 & 3.964 \\
\hline $11 \mathrm{H}-1,94-96$ & 94.55 & 94.65 & 94.75 & 0.119 & 3.819 \\
\hline $11 \mathrm{H}-1,142-144$ & 95.12 & 95.13 & 95.14 & 0.124 & 3.284 \\
\hline $11 \mathrm{H}-2,16-18$ & 95.27 & 95.37 & 95.47 & -0.095 & 3.280 \\
\hline $11 \mathrm{H}-2,94-96$ & 96.05 & 96.15 & 96.25 & 0.273 & 3.754 \\
\hline $11 \mathrm{H}-2,134-136$ & 96.54 & 96.55 & 96.56 & 0.083 & 3.336 \\
\hline $11 \mathrm{H}-3,94-96$ & 97.55 & 97.65 & 97.75 & -0.368 & 2.927 \\
\hline $11 \mathrm{H}-3,136-138$ & 98.06 & 98.07 & 98.08 & -0.368 & 2.987 \\
\hline $11 \mathrm{H}-4,11-13$ & 98.29 & 98.30 & 98.31 & -0.814 & 2.599 \\
\hline $11 \mathrm{H}-4,22-24$ & 98.31 & 98.41 & 98.51 & -0.626 & 3.073 \\
\hline $11 \mathrm{H}-4,40-42$ & 98.58 & 98.59 & 98.60 & -0.696 & 3.229 \\
\hline $11 \mathrm{H}-4,80-82$ & 98.98 & 98.99 & 99.00 & -0.523 & 3.896 \\
\hline $11 \mathrm{H}-4,94-96$ & 99.03 & 99.13 & 99.23 & -0.517 & 3.862 \\
\hline
\end{tabular}


Table T2 (continued). (Continued on next page.)

\begin{tabular}{|c|c|c|c|c|c|}
\hline $\begin{array}{l}\text { Core, section, } \\
\text { interval }(\mathrm{cm})\end{array}$ & $\begin{array}{l}\text { Top depth } \\
\text { CSF-A (m) }\end{array}$ & $\begin{array}{l}\text { Middle depth } \\
\text { CSF-A }(\mathrm{m})\end{array}$ & $\begin{array}{l}\text { Bottom depth } \\
\text { CSF-A (m) }\end{array}$ & $\begin{array}{c}\delta^{13} \mathrm{C} \\
(\% \text { VPDB })\end{array}$ & $\begin{array}{c}\delta^{18} \mathrm{O} \\
(\% \circ \mathrm{VPDB})\end{array}$ \\
\hline $11 \mathrm{H}-4,110-112$ & 99.28 & 99.29 & 99.30 & -0.539 & 3.826 \\
\hline $11 \mathrm{H}-4,142-144$ & 99.60 & 99.61 & 99.62 & -0.276 & 4.237 \\
\hline $11 \mathrm{H}-5,19-21$ & 99.78 & 99.88 & 99.98 & -0.142 & 3.766 \\
\hline $11 \mathrm{H}-5,94-96$ & 100.53 & 100.63 & 100.73 & -0.261 & 3.751 \\
\hline $11 \mathrm{H}-6,19-21$ & 101.28 & 101.38 & 101.48 & -0.236 & 3.827 \\
\hline $11 \mathrm{H}-6,94-96$ & 102.03 & 102.13 & 102.23 & -0.057 & 3.950 \\
\hline $11 \mathrm{H}-7,19-21$ & 102.28 & 102.38 & 102.48 & -0.274 & 3.738 \\
\hline $11 \mathrm{H}-7,92-94$ & 103.01 & 103.11 & 103.21 & -0.198 & 3.890 \\
\hline $12 \mathrm{H}-1,19-21$ & 103.30 & 103.40 & 103.50 & -0.236 & 3.864 \\
\hline $12 \mathrm{H}-1,94-96$ & 104.05 & 104.15 & 104.25 & 0.198 & 3.718 \\
\hline $12 \mathrm{H}-2,19-21$ & 104.80 & 104.90 & 105.00 & 0.097 & 3.996 \\
\hline $12 \mathrm{H}-2,94-96$ & 105.55 & 105.65 & 105.75 & -0.170 & 3.745 \\
\hline $12 \mathrm{H}-3,94-96$ & 107.01 & 107.11 & 107.21 & -0.128 & 3.899 \\
\hline $12 \mathrm{H}-4,19-21$ & 107.76 & 107.86 & 107.96 & -0.119 & 4.041 \\
\hline $12 \mathrm{H}-6,19-21$ & 110.76 & 110.86 & 110.96 & -0.174 & 3.897 \\
\hline $12 \mathrm{H}-6,94-96$ & 111.51 & 111.61 & 111.71 & -0.403 & 3.809 \\
\hline $13 \mathrm{H}-1,19-21$ & 112.80 & 112.90 & 113.00 & 0.243 & 3.569 \\
\hline $13 \mathrm{H}-1,93-95$ & 113.54 & 113.64 & 113.74 & -0.073 & 3.072 \\
\hline $13 \mathrm{H}-2,94-96$ & 115.03 & 115.13 & 115.23 & -0.173 & 3.673 \\
\hline $13 \mathrm{H}-3,19-21$ & 115.78 & 115.88 & 115.98 & -0.042 & 3.934 \\
\hline $13 \mathrm{H}-3,94-96$ & 116.53 & 116.63 & 116.73 & 0.023 & 3.953 \\
\hline $13 \mathrm{H}-4,19-21$ & 117.28 & 117.38 & 117.48 & -0.013 & 4.104 \\
\hline $13 \mathrm{H}-4,94-96$ & 118.03 & 118.13 & 118.23 & -0.299 & 3.930 \\
\hline $13 \mathrm{H}-6,19-21$ & 120.28 & 120.38 & 120.48 & -0.384 & 3.860 \\
\hline $14 \mathrm{H}-1,19-21$ & 122.30 & 122.40 & 122.50 & -0.247 & 3.879 \\
\hline $14 \mathrm{H}-1,94-96$ & 123.14 & 123.15 & 123.16 & -0.323 & 2.973 \\
\hline $14 \mathrm{H}-2,94-96$ & 124.64 & 124.65 & 124.66 & -0.501 & 3.239 \\
\hline $14 \mathrm{H}-3,19-21$ & 125.39 & 125.40 & 125.41 & -0.54 & 3.677 \\
\hline $14 \mathrm{H}-3,94-96$ & 126.05 & 126.15 & 126.25 & -0.037 & 3.712 \\
\hline $14 \mathrm{H}-4,19-21$ & 126.80 & 126.90 & 127.00 & -0.102 & 3.915 \\
\hline $14 \mathrm{H}-5,19-21$ & 128.30 & 128.40 & 128.50 & 0.060 & 3.819 \\
\hline $14 \mathrm{H}-5,94-96$ & 129.05 & 129.15 & 129.25 & -0.168 & 3.779 \\
\hline $14 \mathrm{H}-6,19-21$ & 129.80 & 129.90 & 130.00 & 0.034 & 4.023 \\
\hline $15 \mathrm{H}-1,19-21$ & 131.80 & 131.90 & 132.00 & 0.179 & 3.757 \\
\hline $15 \mathrm{H}-1,84-86$ & 132.45 & 132.55 & 132.65 & 0.123 & 3.757 \\
\hline $15 \mathrm{H}-2,19-21$ & 133.39 & 133.40 & 133.41 & 0.11 & 3.254 \\
\hline $15 \mathrm{H}-2,89-91$ & 134.00 & 134.10 & 134.20 & 0.251 & 3.578 \\
\hline $15 \mathrm{H}-3,19-21$ & 134.82 & 134.83 & 134.84 & -0.162 & 3.059 \\
\hline $15 \mathrm{H}-3,94-96$ & 135.57 & 135.58 & 135.59 & 0.192 & 3.287 \\
\hline $15 \mathrm{H}-4,19-21$ & 136.32 & 136.33 & 136.34 & 0.163 & 3.429 \\
\hline $15 \mathrm{H}-4,94-96$ & 136.98 & 137.08 & 137.18 & 0.129 & 3.852 \\
\hline $15 \mathrm{H}-5,19-21$ & 137.60 & 137.70 & 137.80 & -0.312 & 3.733 \\
\hline $15 \mathrm{H}-5,94-96$ & 138.44 & 138.45 & 138.46 & -0.052 & 3.624 \\
\hline $15 \mathrm{H}-6,19-21$ & 139.19 & 139.20 & 139.21 & -0.058 & 3.368 \\
\hline $15 \mathrm{H}-6,94-96$ & 139.85 & 139.95 & 140.05 & 0.108 & 3.472 \\
\hline 15H-7, 19-21 & 140.69 & 140.70 & 140.71 & 0.211 & 3.33 \\
\hline $16 \mathrm{H}-1,94-96$ & 142.05 & 142.15 & 142.25 & 0.147 & 3.542 \\
\hline $16 \mathrm{H}-2,94-96$ & 143.55 & 143.65 & 143.75 & -0.060 & 3.534 \\
\hline $16 \mathrm{H}-3,14-16$ & 144.31 & 144.32 & 144.33 & 0.125 & 3.019 \\
\hline $16 \mathrm{H}-3,34-36$ & 144.51 & 144.52 & 144.53 & 0.032 & 3.032 \\
\hline $16 \mathrm{H}-3,54-56$ & 144.71 & 144.72 & 144.73 & 0.154 & 3.065 \\
\hline $16 \mathrm{H}-3,74-76$ & 144.91 & 144.92 & 144.93 & -0.161 & 3.279 \\
\hline $16 \mathrm{H}-3,94-96$ & 145.02 & 145.12 & 145.22 & -0.086 & 3.143 \\
\hline $16 \mathrm{H}-3,91-93$ & 145.08 & 145.09 & 145.1 & -0.092 & 3.058 \\
\hline $16 \mathrm{H}-3,114-116$ & 145.31 & 145.32 & 145.33 & -0.265 & 2.647 \\
\hline $16 \mathrm{H}-3,134-136$ & 145.51 & 145.52 & 145.53 & 0.627 & 3.086 \\
\hline $16 \mathrm{H}-4,4-6$ & 145.71 & 145.72 & 145.73 & 0.438 & 3.226 \\
\hline $16 \mathrm{H}-4,24-26$ & 145.91 & 145.92 & 145.93 & 0.035 & 3.107 \\
\hline $16 \mathrm{H}-4,44-46$ & 146.11 & 146.12 & 146.13 & 0.017 & 3.007 \\
\hline $16 \mathrm{H}-4,67-69$ & 146.34 & 146.35 & 146.36 & 0.038 & 2.683 \\
\hline $16 \mathrm{H}-4,84-86$ & 146.51 & 146.52 & 146.53 & 0.362 & 2.674 \\
\hline $16 \mathrm{H}-4,107-109$ & 146.74 & 146.75 & 146.76 & 0.421 & 3.168 \\
\hline $16 \mathrm{H}-4,120-122$ & 146.87 & 146.88 & 146.89 & 0.018 & 3.3 \\
\hline $16 \mathrm{H}-4,144-146$ & 147.11 & 147.12 & 147.13 & 0.559 & 2.58 \\
\hline $16 \mathrm{H}-5,14-16$ & 147.31 & 147.32 & 147.33 & 0.429 & 2.912 \\
\hline $16 \mathrm{H}-5,34-36$ & 147.51 & 147.52 & 147.53 & -0.246 & 3.277 \\
\hline $16 \mathrm{H}-5,54-56$ & 147.71 & 147.72 & 147.73 & -0.296 & 3.343 \\
\hline $16 \mathrm{H}-5,75-77$ & 147.92 & 147.93 & 147.94 & 0.376 & 3.279 \\
\hline $16 \mathrm{H}-5,91-93$ & 148.08 & 148.09 & 148.10 & 0.069 & 3.274 \\
\hline
\end{tabular}


Table T2 (continued). (Continued on next page.)

\begin{tabular}{|c|c|c|c|c|c|}
\hline $\begin{array}{l}\text { Core, section, } \\
\text { interval }(\mathrm{cm})\end{array}$ & $\begin{array}{l}\text { Top depth } \\
\text { CSF-A (m) }\end{array}$ & $\begin{array}{l}\text { Middle depth } \\
\text { CSF-A }(\mathrm{m})\end{array}$ & $\begin{array}{l}\text { Bottom depth } \\
\text { CSF-A (m) }\end{array}$ & $\begin{array}{c}\delta^{13} \mathrm{C} \\
(\% \text { VPDB })\end{array}$ & $\begin{array}{c}\delta^{18} \mathrm{O} \\
(\% \circ \mathrm{VPDB})\end{array}$ \\
\hline $16 \mathrm{H}-5,114-116$ & 148.31 & 148.32 & 148.33 & 0.008 & 3.392 \\
\hline $16 \mathrm{H}-5,134-136$ & 148.51 & 148.52 & 148.53 & -0.182 & 3.434 \\
\hline $16 \mathrm{H}-6,4-6$ & 148.71 & 148.72 & 148.73 & -0.057 & 3.248 \\
\hline $16 \mathrm{H}-6,24-26$ & 148.91 & 148.92 & 148.93 & -0.329 & 3.352 \\
\hline $16 \mathrm{H}-6,44-46$ & 149.11 & 149.12 & 149.13 & 0.167 & 3.338 \\
\hline $16 \mathrm{H}-6,60-62$ & 149.27 & 149.28 & 149.29 & -0.094 & 3.12 \\
\hline $16 \mathrm{H}-6,84-86$ & 149.51 & 149.52 & 149.53 & -0.347 & 3.258 \\
\hline $16 \mathrm{H}-6,93-95$ & 149.51 & 149.61 & 149.71 & -0.251 & 3.553 \\
\hline $16 \mathrm{H}-6,107-109$ & 149.74 & 149.75 & 149.76 & -0.426 & 3.279 \\
\hline $16 \mathrm{H}-6,128-130$ & 149.95 & 149.96 & 149.97 & -0.584 & 3.307 \\
\hline $16 \mathrm{H}-7,7-9$ & 150.11 & 150.12 & 150.13 & -0.189 & 3.347 \\
\hline $16 \mathrm{H}-7,27-29$ & 150.31 & 150.32 & 150.33 & 0.042 & 3.269 \\
\hline $17 \mathrm{H}-1,93-95$ & 151.54 & 151.64 & 151.74 & -0.147 & 3.395 \\
\hline $17 \mathrm{H}-2,94-96$ & 153.05 & 153.15 & 153.25 & -0.550 & 3.022 \\
\hline $17 \mathrm{H}-4,90-92$ & 155.30 & 155.40 & 155.50 & -0.238 & 3.553 \\
\hline $18 \mathrm{H}-1,94-96$ & 157.05 & 157.15 & 157.25 & -0.269 & 2.937 \\
\hline $18 \mathrm{H}-2,92-94$ & 158.53 & 158.63 & 158.73 & -0.148 & 3.216 \\
\hline $18 \mathrm{H}-3,93-95$ & 160.04 & 160.14 & 160.24 & -0.017 & 3.801 \\
\hline $18 \mathrm{H}-5,94-96$ & 161.45 & 161.55 & 161.65 & -0.073 & 3.844 \\
\hline $19 \mathrm{H}-1,95-97$ & 166.56 & 166.66 & 166.76 & -0.501 & 3.790 \\
\hline $19 \mathrm{H}-2,94-96$ & 168.05 & 168.15 & 168.25 & -0.165 & 3.418 \\
\hline $19 \mathrm{H}-4,94-96$ & 171.05 & 171.15 & 171.25 & -0.558 & 3.715 \\
\hline $19 \mathrm{H}-5,19-21$ & 171.80 & 171.90 & 172.00 & -0.216 & 3.730 \\
\hline $19 \mathrm{H}-6,19-21$ & 173.27 & 173.37 & 173.47 & -0.767 & 3.766 \\
\hline $20 \mathrm{H}-1,19-21$ & 174.80 & 174.90 & 175.00 & -0.087 & 3.686 \\
\hline $20 \mathrm{H}-2,19-21$ & 176.30 & 176.40 & 176.50 & 0.113 & 3.698 \\
\hline $20 \mathrm{H}-3,19-21$ & 177.48 & 177.58 & 177.68 & -0.237 & 3.573 \\
\hline $21 \mathrm{H}-2,94-96$ & 182.05 & 182.15 & 182.25 & -0.161 & 3.547 \\
\hline $21 \mathrm{H}-3,19-21$ & 182.80 & 182.90 & 183.00 & -0.220 & 3.242 \\
\hline $21 \mathrm{H}-3,94-96$ & 183.55 & 183.65 & 183.75 & 0.269 & 3.224 \\
\hline $21 \mathrm{H}-5,94-96$ & 186.55 & 186.65 & 186.75 & -0.097 & 3.252 \\
\hline $21 \mathrm{H}-6,94-96$ & 188.05 & 188.15 & 188.25 & 0.001 & 3.542 \\
\hline $22 \mathrm{H}-1,19-21$ & 189.30 & 189.40 & 189.50 & 0.032 & 3.568 \\
\hline $22 \mathrm{H}-1,94-96$ & 190.05 & 190.15 & 190.25 & 0.371 & 3.437 \\
\hline $22 \mathrm{H}-2,19-21$ & 190.80 & 190.90 & 191.00 & 0.101 & 3.189 \\
\hline $22 \mathrm{H}-2,94-96$ & 191.55 & 191.65 & 191.75 & -0.027 & 3.399 \\
\hline $22 \mathrm{H}-3,19-21$ & 192.30 & 192.40 & 192.50 & 0.040 & 3.464 \\
\hline $22 \mathrm{H}-3,93-95$ & 193.04 & 193.14 & 193.24 & -0.281 & 3.379 \\
\hline $22 \mathrm{H}-4,19-21$ & 193.80 & 193.90 & 194.00 & 0.036 & 3.457 \\
\hline $22 \mathrm{H}-5,19-21$ & 195.30 & 195.40 & 195.50 & -0.003 & 3.476 \\
\hline $22 \mathrm{H}-5,95-97$ & 196.06 & 196.16 & 196.26 & -0.371 & 3.427 \\
\hline $22 \mathrm{H}-6,19-21$ & 196.80 & 196.90 & 197.00 & -0.085 & 3.270 \\
\hline $22 \mathrm{H}-6,94-96$ & 197.55 & 197.65 & 197.75 & 0.105 & 3.219 \\
\hline $22 \mathrm{H}-6,98-100$ & 197.68 & 197.69 & 197.70 & -0.071 & 3.069 \\
\hline $22 \mathrm{H}-6,118-120$ & 197.88 & 197.89 & 197.90 & 0.215 & 3.048 \\
\hline $22 \mathrm{H}-6,136-138$ & 198.06 & 198.07 & 198.08 & 0.018 & 2.787 \\
\hline $22 \mathrm{H}-7,9-11$ & 198.29 & 198.30 & 198.31 & -0.008 & 3.058 \\
\hline $22 \mathrm{H}-7,19-21$ & 198.30 & 198.40 & 198.50 & 0.292 & 3.076 \\
\hline $22 \mathrm{H}-7,31-33$ & 198.51 & 198.52 & 198.53 & 0.139 & 2.973 \\
\hline $22 \mathrm{H}-7,49-51$ & 198.69 & 198.70 & 198.71 & 0.083 & 2.838 \\
\hline $23 \mathrm{H}-1,37-39$ & 199.07 & 199.08 & 199.09 & -0.03 & 3.362 \\
\hline $23 \mathrm{H}-1,57-59$ & 199.27 & 199.28 & 199.29 & 0.183 & 3.099 \\
\hline $23 \mathrm{H}-1,78-80$ & 199.48 & 199.49 & 199.50 & 0.346 & 2.837 \\
\hline $23 \mathrm{H}-1,94-96$ & 199.55 & 199.65 & 199.75 & 0.243 & 3.145 \\
\hline $23 \mathrm{H}-1,97-99$ & 199.67 & 199.68 & 199.69 & 0.384 & 3.009 \\
\hline $23 \mathrm{H}-1,122-124$ & 199.92 & 199.93 & 199.94 & -0.112 & 2.578 \\
\hline $23 \mathrm{H}-1,137-139$ & 200.07 & 200.08 & 200.09 & 0.03 & 3.4 \\
\hline $23 \mathrm{H}-2,9-11$ & 200.29 & 200.30 & 200.31 & 0.02 & 3.001 \\
\hline $23 \mathrm{H}-2,19-21$ & 200.30 & 200.40 & 200.50 & 0.099 & 3.235 \\
\hline $23 \mathrm{H}-2,32-34$ & 200.52 & 200.53 & 200.54 & -0.01 & 2.64 \\
\hline $23 \mathrm{H}-2,52-54$ & 200.72 & 200.73 & 200.74 & 0.197 & 3.745 \\
\hline $23 \mathrm{H}-2,69-71$ & 200.89 & 200.90 & 200.91 & -0.019 & 3.754 \\
\hline $23 \mathrm{H}-2,94-96$ & 201.05 & 201.15 & 201.25 & -0.468 & 3.916 \\
\hline $23 \mathrm{H}-2,86-88$ & 201.06 & 201.07 & 201.08 & -0.241 & 3.651 \\
\hline $23 \mathrm{H}-2,129-131$ & 201.49 & 201.50 & 201.51 & -0.138 & 3.593 \\
\hline $23 \mathrm{H}-2,147-149$ & 201.67 & 201.68 & 201.69 & -0.162 & 3.522 \\
\hline $23 \mathrm{H}-3,19-21$ & 201.80 & 201.90 & 202.00 & -0.141 & 3.690 \\
\hline $23 \mathrm{H}-3,22-24$ & 201.92 & 201.93 & 201.94 & -0.215 & 3.652 \\
\hline $23 \mathrm{H}-3,94-96$ & 202.55 & 202.65 & 202.75 & 0.039 & 3.893 \\
\hline
\end{tabular}


Table T2 (continued). (Continued on next page.)

\begin{tabular}{|c|c|c|c|c|c|}
\hline $\begin{array}{l}\text { Core, section, } \\
\text { interval }(\mathrm{cm})\end{array}$ & $\begin{array}{l}\text { Top depth } \\
\text { CSF-A (m) }\end{array}$ & $\begin{array}{l}\text { Middle depth } \\
\text { CSF-A }(\mathrm{m})\end{array}$ & $\begin{array}{l}\text { Bottom depth } \\
\text { CSF-A (m) }\end{array}$ & $\begin{array}{c}\delta^{13} \mathrm{C} \\
(\% \circ \mathrm{VPDB})\end{array}$ & $\begin{array}{c}\delta^{18} \mathrm{O} \\
(\% \circ \mathrm{VPDB})\end{array}$ \\
\hline $23 \mathrm{H}-4,19-21$ & 203.30 & 203.40 & 203.50 & 0.131 & 3.808 \\
\hline $23 \mathrm{H}-4,95-97$ & 204.06 & 204.16 & 204.26 & -0.028 & 3.798 \\
\hline $23 \mathrm{H}-5,19-21$ & 204.80 & 204.90 & 205.00 & 0.041 & 3.791 \\
\hline $23 \mathrm{H}-5,94-96$ & 205.55 & 205.65 & 205.75 & 0.245 & 3.644 \\
\hline $23 \mathrm{H}-6,19-21$ & 206.30 & 206.40 & 206.50 & -0.061 & 3.489 \\
\hline $23 \mathrm{H}-6,94-96$ & 207.05 & 207.15 & 207.25 & -0.107 & 3.295 \\
\hline $23 \mathrm{H}-7,19-21$ & 207.80 & 207.90 & 208.00 & 0.289 & 3.281 \\
\hline $24 \mathrm{H}-1,19-21$ & 208.30 & 208.40 & 208.50 & 0.144 & 3.609 \\
\hline $24 \mathrm{H}-1,94-96$ & 209.05 & 209.15 & 209.25 & -0.124 & 3.646 \\
\hline $24 \mathrm{H}-2,19-21$ & 209.80 & 209.90 & 210.00 & 0.317 & 3.687 \\
\hline $24 \mathrm{H}-2,93-95$ & 210.54 & 210.64 & 210.74 & -0.313 & 3.663 \\
\hline $24 \mathrm{H}-3,19-21$ & 211.30 & 211.40 & 211.50 & -0.171 & 3.568 \\
\hline $24 \mathrm{H}-3,94-96$ & 212.05 & 212.15 & 212.25 & -0.546 & 3.670 \\
\hline $24 \mathrm{H}-4,19-21$ & 212.80 & 212.90 & 213.00 & -0.106 & 3.506 \\
\hline $24 \mathrm{H}-4,94-96$ & 213.55 & 213.65 & 213.75 & -0.089 & 3.522 \\
\hline $24 \mathrm{H}-5,18-20$ & 214.29 & 214.39 & 214.49 & 0.005 & 3.619 \\
\hline $24 \mathrm{H}-5,94-96$ & 215.05 & 215.15 & 215.25 & -0.608 & 3.529 \\
\hline $24 \mathrm{H}-6,94-96$ & 216.55 & 216.65 & 216.75 & -0.491 & 3.525 \\
\hline $24 \mathrm{H}-7,18-20$ & 217.29 & 217.39 & 217.49 & -0.382 & 3.537 \\
\hline $25 \mathrm{H}-1,19-21$ & 217.80 & 217.90 & 218.00 & -0.427 & 4.089 \\
\hline $25 \mathrm{H}-1,94-96$ & 218.55 & 218.65 & 218.75 & -0.411 & 4.138 \\
\hline $25 \mathrm{H}-3,94-96$ & 221.55 & 221.65 & 221.75 & 0.248 & 3.849 \\
\hline $26 \mathrm{H}-2,19-21$ & 228.72 & 228.82 & 228.92 & -0.230 & 3.551 \\
\hline $26 \mathrm{H}-2,94-96$ & 229.47 & 229.57 & 229.67 & -0.196 & 3.558 \\
\hline $26 \mathrm{H}-3,95-97$ & 230.98 & 231.08 & 231.18 & -0.141 & 3.549 \\
\hline 27H-1, 94-96 & 237.55 & 237.65 & 237.75 & 0.002 & 3.317 \\
\hline 27H-5, 94-96 & 243.55 & 243.65 & 243.75 & 0.004 & 3.118 \\
\hline $27 \mathrm{H}-6,19-21$ & 244.30 & 244.40 & 244.50 & -0.165 & 2.999 \\
\hline $27 \mathrm{H}-6,64-66$ & 244.75 & 244.85 & 244.95 & -0.148 & 2.696 \\
\hline 27H-7, 19-21 & 245.30 & 245.40 & 245.50 & -0.013 & 2.619 \\
\hline $28 \mathrm{H}-1,19-21$ & 246.30 & 246.40 & 246.50 & -0.201 & 3.274 \\
\hline $28 \mathrm{H}-1,94-96$ & 247.05 & 247.15 & 247.25 & -0.160 & 3.768 \\
\hline $28 \mathrm{H}-2,4-6$ & 247.67 & 247.68 & 247.69 & -0.403 & 3.749 \\
\hline $28 \mathrm{H}-2,19-21$ & 247.73 & 247.83 & 247.93 & -0.173 & 3.810 \\
\hline $28 \mathrm{H}-2,23-25$ & 247.86 & 247.87 & 247.88 & -0.095 & 3.478 \\
\hline $28 \mathrm{H}-2,44-46$ & 248.07 & 248.08 & 248.09 & -0.479 & 3.609 \\
\hline $28 \mathrm{H}-2,68-70$ & 248.31 & 248.32 & 248.33 & -0.204 & 3.418 \\
\hline $28 \mathrm{H}-2,84-86$ & 248.47 & 248.48 & 248.49 & -0.38 & 3.339 \\
\hline $28 \mathrm{H}-2,94-96$ & 248.48 & 248.58 & 248.68 & 0.065 & 3.603 \\
\hline $28 \mathrm{H}-2,102-104$ & 248.65 & 248.66 & 248.67 & -0.03 & 3.28 \\
\hline $28 \mathrm{H}-2,124-126$ & 248.87 & 248.88 & 248.89 & 0.074 & 3.099 \\
\hline $28 \mathrm{H}-3,19-21$ & 249.23 & 249.33 & 249.43 & -0.306 & 3.070 \\
\hline $28 \mathrm{H}-3,15-17$ & 249.28 & 249.29 & 249.30 & 0.019 & 3.027 \\
\hline $28 \mathrm{H}-3,34-36$ & 249.47 & 249.48 & 249.49 & -0.167 & 2.627 \\
\hline $28 \mathrm{H}-3,54-56$ & 249.67 & 249.68 & 249.69 & -0.572 & 2.738 \\
\hline $28 \mathrm{H}-3,73-75$ & 249.86 & 249.87 & 249.88 & -0.46 & 2.706 \\
\hline $28 \mathrm{H}-3,94-96$ & 249.98 & 250.08 & 250.18 & -0.390 & 3.011 \\
\hline $28 \mathrm{H}-3,97-99$ & 250.10 & 250.11 & 250.12 & -0.356 & 3.059 \\
\hline $28 \mathrm{H}-4,17-19$ & 250.21 & 250.31 & 250.41 & -0.694 & 3.856 \\
\hline $28 \mathrm{H}-4,14-16$ & 250.27 & 250.28 & 250.29 & -0.694 & 3.628 \\
\hline $28 \mathrm{H}-4,33-35$ & 250.46 & 250.47 & 250.48 & -0.662 & 3.638 \\
\hline $28 \mathrm{H}-4,51-53$ & 250.64 & 250.65 & 250.66 & -0.749 & 3.387 \\
\hline $29 \mathrm{H}-1,27-29$ & 251.47 & 251.48 & 251.49 & -0.852 & 3.538 \\
\hline $29 \mathrm{H}-1,47-49$ & 251.67 & 251.68 & 251.69 & -1.103 & 3.592 \\
\hline $29 \mathrm{H}-1,69-71$ & 251.90 & 251.91 & 251.92 & -0.771 & 3.687 \\
\hline $29 \mathrm{H}-1,94-96$ & 252.05 & 252.15 & 252.25 & -0.875 & 3.796 \\
\hline 29H-1, 129-131 & 252.49 & 252.50 & 252.51 & -0.843 & 3.545 \\
\hline $29 \mathrm{H}-1,146-148$ & 252.66 & 252.67 & 252.68 & -1.13 & 3.694 \\
\hline $29 \mathrm{H}-2,19-21$ & 252.80 & 252.90 & 253.00 & -1.025 & 3.544 \\
\hline $29 \mathrm{H}-2,15-17$ & 252.85 & 252.86 & 252.87 & -0.539 & 3.678 \\
\hline $29 \mathrm{H}-2,94-96$ & 253.55 & 253.65 & 253.75 & -0.802 & 3.821 \\
\hline $29 \mathrm{H}-3,19-21$ & 254.30 & 254.40 & 254.50 & -0.268 & 3.441 \\
\hline $29 \mathrm{H}-3,94-96$ & 255.05 & 255.15 & 255.25 & -1.241 & 3.472 \\
\hline $30 \mathrm{H}-1,17-19$ & 257.78 & 257.88 & 257.98 & -1.066 & 3.333 \\
\hline $30 \mathrm{H}-2,94-96$ & 260.05 & 260.15 & 260.25 & -1.011 & 3.340 \\
\hline $30 \mathrm{H}-3,19-21$ & 260.80 & 260.90 & 261.00 & -0.808 & 3.828 \\
\hline $30 \mathrm{H}-4,18-20$ & 262.29 & 262.39 & 262.49 & -1.389 & 3.506 \\
\hline $31 \mathrm{H}-2,19-21$ & 268.80 & 268.90 & 269.00 & -0.462 & 3.821 \\
\hline $31 \mathrm{H}-5,18-20$ & 271.10 & 271.20 & 271.30 & -0.963 & 3.470 \\
\hline
\end{tabular}


Table T2 (continued). (Continued on next page.)

\begin{tabular}{|c|c|c|c|c|c|}
\hline $\begin{array}{l}\text { Core, section, } \\
\text { interval }(\mathrm{cm})\end{array}$ & $\begin{array}{l}\text { Top depth } \\
\text { CSF-A (m) }\end{array}$ & $\begin{array}{l}\text { Middle depth } \\
\text { CSF-A (m) }\end{array}$ & $\begin{array}{l}\text { Bottom depth } \\
\text { CSF-A (m) }\end{array}$ & $\begin{array}{c}\delta^{13} \mathrm{C} \\
(\% \circ \mathrm{VPDB})\end{array}$ & $\begin{array}{c}\delta^{18} \mathrm{O} \\
(\% \circ \mathrm{VPDB})\end{array}$ \\
\hline $32 \mathrm{H}-1,19-22$ & 272.31 & 272.41 & 272.51 & -0.512 & 3.815 \\
\hline $32 \mathrm{H}-3,19-21$ & 275.30 & 275.40 & 275.50 & -0.628 & 3.404 \\
\hline $32 \mathrm{H}-4,19-21$ & 276.80 & 276.90 & 277.00 & -0.503 & 3.091 \\
\hline $32 \mathrm{H}-4,94-96$ & 277.55 & 277.65 & 277.75 & -0.500 & 3.183 \\
\hline $32 \mathrm{H}-5,19-21$ & 278.25 & 278.35 & 278.45 & -0.089 & 3.061 \\
\hline $33 \mathrm{H}-1,19-21$ & 281.80 & 281.90 & 282.00 & 0.083 & 2.897 \\
\hline $33 \mathrm{H}-1,93-95$ & 282.54 & 282.64 & 282.74 & -0.233 & 3.254 \\
\hline $33 \mathrm{H}-2,19-21$ & 283.21 & 283.31 & 283.41 & -0.172 & 3.481 \\
\hline $33 \mathrm{H}-3,19-21$ & 283.98 & 284.08 & 284.18 & -0.248 & 3.297 \\
\hline $33 \mathrm{H}-4,94-96$ & 285.49 & 285.59 & 285.69 & -0.416 & 3.287 \\
\hline $33 \mathrm{H}-5,19-21$ & 286.24 & 286.34 & 286.44 & -0.407 & 3.371 \\
\hline $33 \mathrm{H}-6,19-21$ & 287.72 & 287.82 & 287.92 & -0.563 & 3.280 \\
\hline $34 \mathrm{H}-2,19-21$ & 288.56 & 288.66 & 288.76 & -0.693 & 3.252 \\
\hline $34 \mathrm{H}-3,18-20$ & 289.98 & 290.08 & 290.18 & -0.147 & 3.147 \\
\hline $34 \mathrm{H}-4,23-25$ & 290.91 & 291.01 & 291.11 & -0.450 & 3.188 \\
\hline $34 \mathrm{H}-5,97-99$ & 292.02 & 292.12 & 292.22 & -0.448 & 3.314 \\
\hline $35 \mathrm{H}-2,19-21$ & 294.44 & 294.54 & 294.64 & 0.140 & 2.710 \\
\hline $36 \mathrm{H}-1,19-21$ & 295.40 & 295.50 & 295.60 & -0.023 & 2.966 \\
\hline $36 \mathrm{H}-2,19-21$ & 296.60 & 296.70 & 296.80 & 0.296 & 3.137 \\
\hline $37 X-1,94-96$ & 297.85 & 297.95 & 298.05 & 0.767 & 2.035 \\
\hline $37 X-2,19-21$ & 298.60 & 298.70 & 298.80 & 0.035 & 3.043 \\
\hline $37 X-C C, 19-21$ & 299.65 & 299.75 & 299.85 & -0.184 & 3.556 \\
\hline $38 \times-1,19-21$ & 302.70 & 302.80 & 302.90 & -0.487 & 3.635 \\
\hline $38 X-2,19-21$ & 304.20 & 304.30 & 304.40 & -0.217 & 3.553 \\
\hline $38 X-2,94-96$ & 304.95 & 305.05 & 305.15 & -0.212 & 3.223 \\
\hline $38 X-3,19-21$ & 305.70 & 305.80 & 305.90 & -0.426 & 3.782 \\
\hline $38 X-4,19-21$ & 307.20 & 307.30 & 307.40 & -0.666 & 3.616 \\
\hline $38 X-6,19-21$ & 310.20 & 310.30 & 310.40 & -0.683 & 3.434 \\
\hline $39 X-1,19-21$ & 312.30 & 312.40 & 312.50 & -0.407 & 3.446 \\
\hline $39 \times-2,19-21$ & 313.80 & 313.90 & 314.00 & -0.673 & 3.313 \\
\hline $39 X-3,19-21$ & 315.30 & 315.40 & 315.50 & -0.760 & 3.229 \\
\hline $39 X-4,19-21$ & 316.80 & 316.90 & 317.00 & 0.066 & 3.390 \\
\hline $39 X-4,94-96$ & 317.55 & 317.65 & 317.75 & -0.563 & 3.368 \\
\hline $39 \times-5,19-21$ & 318.30 & 318.40 & 318.50 & -0.652 & 3.341 \\
\hline $39 \times-6,19-21$ & 319.80 & 319.90 & 320.00 & -0.656 & 2.999 \\
\hline $39 X-7,19-21$ & 321.10 & 321.20 & 321.30 & 0.323 & 3.235 \\
\hline $40 X-1,94-96$ & 322.75 & 322.85 & 322.95 & 0.022 & 3.261 \\
\hline $40 X-2,19-21$ & 323.50 & 323.60 & 323.70 & -0.450 & 3.383 \\
\hline $40 X-3,19-21$ & 325.00 & 325.10 & 325.20 & -0.275 & 2.864 \\
\hline $40 \times-4,19-21$ & 326.50 & 326.60 & 326.70 & -0.286 & 3.156 \\
\hline $40 X-4,94-96$ & 327.25 & 327.35 & 327.45 & -0.243 & 3.215 \\
\hline $40 X-5,94-96$ & 328.75 & 328.85 & 328.95 & -0.119 & 2.880 \\
\hline $40 X-6,94-96$ & 330.25 & 330.35 & 330.45 & 0.161 & 2.607 \\
\hline $41 X-1,19-21$ & 331.60 & 331.70 & 331.80 & -0.207 & 3.258 \\
\hline $41 X-1,94-96$ & 332.35 & 332.45 & 332.55 & -0.328 & 3.429 \\
\hline $41 X-2,94-96$ & 333.85 & 333.95 & 334.05 & -0.600 & 3.595 \\
\hline $41 X-3,94-96$ & 335.35 & 335.45 & 335.55 & -0.141 & 3.389 \\
\hline $41 X-4,19-21$ & 336.10 & 336.20 & 336.30 & -0.222 & 3.521 \\
\hline $41 X-4,94-96$ & 336.85 & 336.95 & 337.05 & -0.278 & 3.530 \\
\hline $41 X-5,19-21$ & 337.60 & 337.70 & 337.80 & -0.239 & 3.346 \\
\hline $41 X-5,94-96$ & 338.35 & 338.45 & 338.55 & -0.423 & 3.308 \\
\hline $41 X-6,94-96$ & 339.85 & 339.95 & 340.05 & -0.326 & 3.273 \\
\hline $42 X-2,19-21$ & 342.70 & 342.80 & 342.90 & -0.377 & 3.524 \\
\hline $42 X-2,94-96$ & 343.45 & 343.55 & 343.65 & -0.610 & 3.690 \\
\hline $42 X-3,19-21$ & 344.20 & 344.30 & 344.40 & -0.501 & 3.385 \\
\hline $42 X-3,94-96$ & 344.95 & 345.05 & 345.15 & -0.472 & 3.187 \\
\hline $42 X-4,19-21$ & 345.70 & 345.80 & 345.90 & -0.439 & 2.940 \\
\hline $42 X-5,18-20$ & 347.19 & 347.29 & 347.39 & 0.837 & 1.345 \\
\hline $42 X-5,94-96$ & 347.95 & 348.05 & 348.15 & 0.092 & 3.008 \\
\hline $43 X-1,20-22$ & 350.81 & 350.91 & 351.01 & -0.962 & 3.950 \\
\hline $43 X-1,95-97$ & 351.56 & 351.66 & 351.76 & -0.836 & 4.028 \\
\hline $43 X-2,19-21$ & 352.30 & 352.40 & 352.50 & -1.254 & 3.828 \\
\hline $43 X-3,19-21$ & 353.80 & 353.90 & 354.00 & -0.560 & 3.679 \\
\hline $43 X-3,94-96$ & 354.55 & 354.65 & 354.75 & -0.152 & 3.230 \\
\hline $43 X-4,19-21$ & 355.30 & 355.40 & 355.50 & 0.021 & 2.753 \\
\hline $43 X-4,94-96$ & 356.05 & 356.15 & 356.25 & -0.010 & 3.324 \\
\hline $43 X-5,94-96$ & 357.55 & 357.65 & 357.75 & 0.521 & 2.977 \\
\hline $43 X-6,94-96$ & 359.05 & 359.15 & 359.25 & 0.092 & 3.282 \\
\hline $44 X-1,19-21$ & 360.50 & 360.60 & 360.70 & -0.106 & 3.535 \\
\hline
\end{tabular}


Table T2 (continued). (Continued on next page.)

\begin{tabular}{|c|c|c|c|c|c|}
\hline $\begin{array}{l}\text { Core, section, } \\
\text { interval }(\mathrm{cm})\end{array}$ & $\begin{array}{l}\text { Top depth } \\
\text { CSF-A (m) }\end{array}$ & $\begin{array}{l}\text { Middle depth } \\
\text { CSF-A (m) }\end{array}$ & $\begin{array}{l}\text { Bottom depth } \\
\text { CSF-A (m) }\end{array}$ & $\begin{array}{c}\delta^{13} \mathrm{C} \\
(\% \circ \mathrm{VPDB})\end{array}$ & $\begin{array}{c}\delta^{18} \mathrm{O} \\
(\% \circ \mathrm{VPDB})\end{array}$ \\
\hline $44 X-1,94-96$ & 361.25 & 361.35 & 361.45 & -0.257 & 3.365 \\
\hline $44 X-2,94-96$ & 362.75 & 362.85 & 362.95 & 0.178 & 3.081 \\
\hline $44 X-3,19-21$ & 363.50 & 363.60 & 363.70 & -0.312 & 2.876 \\
\hline $44 X-4,19-21$ & 365.00 & 365.10 & 365.20 & -0.060 & 2.854 \\
\hline $44 X-5,19-21$ & 366.50 & 366.60 & 366.70 & 0.189 & 3.239 \\
\hline $44 X-5,94-96$ & 367.25 & 367.35 & 367.45 & 0.036 & 3.281 \\
\hline $45 X-1,19-21$ & 370.10 & 370.20 & 370.30 & -0.296 & 3.392 \\
\hline $45 X-2,19-21$ & 371.60 & 371.70 & 371.80 & -0.340 & 3.432 \\
\hline $45 X-2,94-96$ & 372.35 & 372.45 & 372.55 & -0.319 & 3.318 \\
\hline $45 X-3,94-96$ & 373.85 & 373.95 & 374.05 & 0.002 & 3.134 \\
\hline $45 X-4,94-96$ & 375.35 & 375.45 & 375.55 & -0.100 & 2.899 \\
\hline $45 X-5,19-21$ & 376.10 & 376.20 & 376.30 & 0.045 & 3.628 \\
\hline $45 X-6,19-21$ & 377.60 & 377.70 & 377.80 & -0.540 & 3.141 \\
\hline $45 X-6,94-96$ & 378.35 & 378.45 & 378.55 & -0.279 & 3.272 \\
\hline $46 X-1,19-21$ & 379.70 & 379.80 & 379.90 & -0.274 & 3.560 \\
\hline $46 X-1,94-96$ & 380.45 & 380.55 & 380.65 & -0.446 & 3.204 \\
\hline $46 X-3,19-21$ & 382.68 & 382.78 & 382.88 & -0.243 & 3.046 \\
\hline $46 X-3,94-96$ & 383.43 & 383.53 & 383.63 & -0.302 & 3.247 \\
\hline $46 X-4,94-96$ & 384.93 & 385.03 & 385.13 & -0.240 & 3.106 \\
\hline $46 X-5,94-96$ & 386.43 & 386.53 & 386.63 & -0.426 & 3.193 \\
\hline $46 X-6,19-21$ & 387.18 & 387.28 & 387.38 & -0.900 & 3.245 \\
\hline $47 X-1,18-20$ & 389.29 & 389.39 & 389.49 & -0.132 & 3.183 \\
\hline $47 X-2,19-21$ & 390.80 & 390.90 & 391.00 & -0.108 & 2.967 \\
\hline $47 X-2,93-95$ & 391.54 & 391.64 & 391.74 & 0.349 & 2.217 \\
\hline $47 X-3,19-21$ & 392.30 & 392.40 & 392.50 & -0.269 & 3.018 \\
\hline $47 X-4,18-20$ & 393.79 & 393.89 & 393.99 & -0.406 & 3.156 \\
\hline $47 X-5,18-20$ & 395.29 & 395.39 & 395.49 & -0.074 & 3.096 \\
\hline $47 X-6,18-20$ & 396.79 & 396.89 & 396.99 & -0.591 & 2.883 \\
\hline $47 X-6,93-95$ & 397.54 & 397.64 & 397.74 & -1.125 & 3.230 \\
\hline $48 X-1,19-21$ & 398.80 & 398.90 & 399.00 & -0.772 & 3.286 \\
\hline $48 X-1,94-96$ & 399.55 & 399.65 & 399.75 & -0.331 & 3.223 \\
\hline $48 X-2,94-96$ & 401.05 & 401.15 & 401.25 & -0.882 & 3.401 \\
\hline $48 X-3,94-96$ & 402.55 & 402.65 & 402.75 & -0.351 & 2.993 \\
\hline $48 \times-4,19-21$ & 403.30 & 403.40 & 403.50 & -0.161 & 2.806 \\
\hline $48 X-5,19-21$ & 404.80 & 404.90 & 405.00 & -0.404 & 3.140 \\
\hline $48 X-7,19-21$ & 407.60 & 407.70 & 407.80 & -0.936 & 1.957 \\
\hline $49 X-1,19-21$ & 408.40 & 408.50 & 408.60 & -0.419 & 3.168 \\
\hline $49 X-2,19-21$ & 409.90 & 410.00 & 410.10 & -0.059 & 3.013 \\
\hline $49 X-3,93-95$ & 412.14 & 412.24 & 412.34 & -0.214 & 2.224 \\
\hline $50 \times-1,19-21$ & 418.00 & 418.10 & 418.20 & -0.012 & 2.741 \\
\hline $50 X-2,19-21$ & 419.50 & 419.60 & 419.70 & -0.611 & 2.930 \\
\hline $50 X-2,98-100$ & 420.29 & 420.39 & 420.49 & 0.033 & 3.098 \\
\hline $50 X-3,94-96$ & 421.75 & 421.85 & 421.95 & 0.138 & 3.041 \\
\hline $50 \times-4,19-21$ & 422.50 & 422.60 & 422.70 & 0.520 & 1.682 \\
\hline $50 \times-5,19-21$ & 424.00 & 424.10 & 424.20 & -0.195 & 3.348 \\
\hline $50 X-6,19-21$ & 425.50 & 425.60 & 425.70 & -0.450 & 2.721 \\
\hline $50 X-7,19-21$ & 426.80 & 426.90 & 427.00 & 0.527 & 2.925 \\
\hline $50 X-7,16-18$ & 426.86 & 426.87 & 426.88 & -0.012 & 2.957 \\
\hline $50 X-7,38-40$ & 427.08 & 427.09 & 427.10 & -0.242 & 3.317 \\
\hline $50 X-C C, 1-3$ & 427.35 & 427.36 & 427.37 & 0.031 & 2.603 \\
\hline $51 X-1,6-8$ & 427.56 & 427.57 & 427.58 & -0.07 & 2.437 \\
\hline $51 X-1,19-21$ & 427.60 & 427.70 & 427.80 & 0.087 & 1.903 \\
\hline $51 X-1,16-18$ & 427.66 & 427.67 & 427.68 & -0.157 & 2.474 \\
\hline $51 X-1,37-39$ & 427.87 & 427.88 & 427.89 & 0.418 & 2.487 \\
\hline $51 X-1,57-59$ & 428.07 & 428.08 & 428.09 & -0.16 & 2.567 \\
\hline $51 X-1,77-79$ & 428.27 & 428.28 & 428.29 & -0.359 & 3.036 \\
\hline $51 X-1,95-97$ & 428.36 & 428.46 & 428.56 & -0.140 & 3.127 \\
\hline $51 X-1,98-100$ & 428.48 & 428.49 & 428.50 & -0.152 & 2.862 \\
\hline $51 X-1,117-119$ & 428.67 & 428.68 & 428.69 & 0.158 & 3.125 \\
\hline $51 X-1,137-139$ & 428.87 & 428.88 & 428.89 & -0.34 & 3.122 \\
\hline $51 X-2,9-11$ & 429.09 & 429.10 & 429.11 & -0.368 & 3.051 \\
\hline $51 X-2,25-27$ & 429.25 & 429.26 & 429.27 & -0.3 & 3.411 \\
\hline $51 X-2,45-47$ & 429.45 & 429.46 & 429.47 & -0.445 & 3.296 \\
\hline $51 X-2,67-69$ & 429.67 & 429.68 & 429.69 & -0.658 & 3.595 \\
\hline $51 X-2,85-87$ & 429.85 & 429.86 & 429.87 & -0.395 & 3.542 \\
\hline $51 X-2,95-97$ & 429.86 & 429.96 & 430.06 & -0.718 & 3.734 \\
\hline $51 X-2,109-111$ & 430.09 & 430.10 & 430.11 & -0.637 & 3.567 \\
\hline $51 X-2,129-131$ & 430.29 & 430.30 & 430.31 & -0.878 & 3.517 \\
\hline $51 \times-2,147-149$ & 430.47 & 430.48 & 430.49 & -0.709 & 3.228 \\
\hline
\end{tabular}


Table T2 (continued). (Continued on next page.)

\begin{tabular}{|c|c|c|c|c|c|}
\hline $\begin{array}{l}\text { Core, section, } \\
\text { interval }(\mathrm{cm})\end{array}$ & $\begin{array}{l}\text { Top depth } \\
\text { CSF-A (m) }\end{array}$ & $\begin{array}{l}\text { Middle depth } \\
\text { CSF-A (m) }\end{array}$ & $\begin{array}{l}\text { Bottom depth } \\
\text { CSF-A (m) }\end{array}$ & $\begin{array}{c}\delta^{13} \mathrm{C} \\
(\% \circ \mathrm{VPDB})\end{array}$ & $\begin{array}{c}\delta^{18} \mathrm{O} \\
(\% \circ \mathrm{VPDB})\end{array}$ \\
\hline $51 X-3,15-17$ & 430.65 & 430.66 & 430.67 & -0.704 & 3.147 \\
\hline $51 X-3,38-40$ & 430.88 & 430.89 & 430.90 & -0.536 & 3.637 \\
\hline $51 X-3,58-60$ & 431.08 & 431.09 & 431.10 & -0.486 & 3.695 \\
\hline $51 X-3,95-97$ & 431.36 & 431.46 & 431.56 & -0.400 & 3.924 \\
\hline $51 X-4,94-96$ & 432.85 & 432.95 & 433.05 & -0.852 & 3.489 \\
\hline $51 X-5,95-97$ & 434.36 & 434.46 & 434.56 & -0.467 & 3.906 \\
\hline $51 X-6,19-21$ & 435.10 & 435.20 & 435.30 & -1.066 & 3.488 \\
\hline $51 X-7,19-21$ & 436.30 & 436.40 & 436.50 & -0.689 & 3.620 \\
\hline $52 X-1,19-21$ & 437.20 & 437.30 & 437.40 & -0.286 & 3.679 \\
\hline $52 X-2,19-21$ & 438.70 & 438.80 & 438.90 & -0.467 & 3.348 \\
\hline $52 X-3,19-21$ & 440.20 & 440.30 & 440.40 & -0.296 & 3.397 \\
\hline $52 X-4,19-21$ & 441.70 & 441.80 & 441.90 & -0.726 & 2.957 \\
\hline $52 X-4,94-96$ & 442.45 & 442.55 & 442.65 & -0.380 & 3.157 \\
\hline $52 X-5,93-95$ & 443.94 & 444.04 & 444.14 & 0.167 & 1.882 \\
\hline $52 X-6,94-96$ & 445.45 & 445.55 & 445.65 & -0.213 & 2.647 \\
\hline $53 X-1,19-21$ & 446.80 & 446.90 & 447.00 & -0.001 & 2.749 \\
\hline $53 X-1,26-28$ & 446.96 & 446.97 & 446.98 & 0.235 & 1.683 \\
\hline $53 X-1,46-48$ & 447.16 & 447.17 & 447.18 & 0.485 & 1.925 \\
\hline $53 X-1,72-74$ & 447.42 & 447.43 & 447.44 & 0.263 & 2.127 \\
\hline $53 X-1,95-97$ & 447.56 & 447.66 & 447.76 & 0.442 & 2.004 \\
\hline $53 X-1,91-93$ & 447.61 & 447.62 & 447.63 & 0.256 & 1.786 \\
\hline $53 X-1,131-133$ & 448.01 & 448.02 & 448.03 & 0.201 & 2.66 \\
\hline $53 X-2,2-4$ & 448.22 & 448.23 & 448.24 & -0.185 & 2.589 \\
\hline $53 X-2,16-18$ & 448.36 & 448.37 & 448.38 & 0.306 & 2.103 \\
\hline $53 X-2,38-40$ & 448.58 & 448.59 & 448.60 & 0.357 & 1.847 \\
\hline $53 X-2,59-61$ & 448.79 & 448.8 & 448.81 & 0.548 & 1.403 \\
\hline $53 \times-2,77-79$ & 448.97 & 448.98 & 448.99 & 0.352 & 1.833 \\
\hline $53 X-2,94-96$ & 449.05 & 449.15 & 449.25 & 0.071 & 1.953 \\
\hline $53 \times-2,97-99$ & 449.17 & 449.18 & 449.19 & 0.415 & 2.423 \\
\hline $53 X-2,117-119$ & 449.37 & 449.38 & 449.39 & -0.413 & 2.04 \\
\hline $53 X-2,137-139$ & 449.57 & 449.58 & 449.59 & 0.319 & 1.632 \\
\hline $53 X-3,8-10$ & 449.78 & 449.79 & 449.80 & 0.139 & 1.252 \\
\hline $53 \times-3,27-29$ & 449.97 & 449.98 & 449.99 & 0.356 & 2.191 \\
\hline $53 X-3,46-48$ & 450.16 & 450.17 & 450.18 & 0.03 & 1.583 \\
\hline $53 X-3,68-70$ & 450.38 & 450.39 & 450.40 & 0.264 & 1.767 \\
\hline $53 X-3,94-96$ & 450.55 & 450.65 & 450.75 & 0.760 & 1.388 \\
\hline $53 X-3,87-89$ & 450.57 & 450.58 & 450.59 & -0.393 & 1.317 \\
\hline $53 X-3,107-109$ & 450.77 & 450.78 & 450.79 & -0.127 & 1.172 \\
\hline $53 X-3,132-134$ & 451.02 & 451.03 & 451.04 & 0.053 & 2.2 \\
\hline $53 X-4,4-6$ & 451.24 & 451.25 & 451.26 & -0.056 & 2.504 \\
\hline $53 \times-4,19-21$ & 451.30 & 451.40 & 451.50 & -0.115 & 2.605 \\
\hline $53 X-4,22-24$ & 451.42 & 451.43 & 451.44 & -0.023 & 2.615 \\
\hline $53 X-4,43-45$ & 451.63 & 451.64 & 451.65 & -0.165 & 2.778 \\
\hline $53 X-4,61-63$ & 451.81 & 451.82 & 451.83 & -0.094 & 2.778 \\
\hline $53 X-4,81-83$ & 452.01 & 452.02 & 452.03 & 0.043 & 1.853 \\
\hline $53 X-4,103-105$ & 452.23 & 452.24 & 452.25 & 0.04 & 2.155 \\
\hline $53 X-4,121-123$ & 452.41 & 452.42 & 452.43 & 0.138 & 2.303 \\
\hline $53 X-4,141-143$ & 452.61 & 452.62 & 452.63 & 0.382 & 1.769 \\
\hline $53 \times-5,7-9$ & 452.77 & 452.78 & 452.79 & -0.021 & 2.145 \\
\hline $53 X-5,19-21$ & 452.80 & 452.90 & 453.00 & -0.160 & 2.641 \\
\hline $53 X-5,27-29$ & 452.97 & 452.98 & 452.99 & 0.13 & 2.57 \\
\hline $53 X-5,52-54$ & 453.22 & 453.23 & 453.24 & -0.618 & 2.88 \\
\hline $53 X-5,71-73$ & 453.41 & 453.42 & 453.43 & -0.292 & 3.05 \\
\hline $53 X-5,94-96$ & 453.55 & 453.65 & 453.75 & -0.262 & 3.383 \\
\hline $53 X-5,97-99$ & 453.67 & 453.68 & 453.69 & -0.332 & 3.203 \\
\hline $53 X-5,113-115$ & 453.83 & 453.84 & 453.85 & -0.521 & 3.055 \\
\hline $53 X-5,131-133$ & 454.01 & 454.02 & 454.03 & -0.583 & 3.104 \\
\hline $53 X-6,2-4$ & 454.19 & 454.20 & 454.21 & -0.429 & 3.152 \\
\hline $53 \times-6,15-17$ & 454.32 & 454.33 & 454.34 & -0.615 & 3.065 \\
\hline $53 X-6,35-37$ & 454.52 & 454.53 & 454.54 & -0.052 & 3.434 \\
\hline $53 X-6,59-61$ & 454.76 & 454.77 & 454.78 & -0.295 & 3.291 \\
\hline $53 X-6,82-84$ & 454.99 & 455.00 & 455.01 & -0.655 & 2.93 \\
\hline $53 X-6,95-97$ & 455.03 & 455.13 & 455.23 & -0.848 & 3.474 \\
\hline $53 X-6,101-103$ & 455.18 & 455.19 & 455.20 & -1.026 & 3.109 \\
\hline $53 X-6,123-125$ & 455.40 & 455.41 & 455.42 & -0.484 & 3.18 \\
\hline $54 X-1,19-21$ & 456.40 & 456.50 & 456.60 & -1.060 & 3.248 \\
\hline $54 X-1,94-96$ & 457.15 & 457.25 & 457.35 & -0.895 & 3.335 \\
\hline $54 X-2,94-96$ & 458.65 & 458.75 & 458.85 & -0.437 & 2.588 \\
\hline
\end{tabular}


Table T2 (continued).

\begin{tabular}{|c|c|c|c|c|c|}
\hline $\begin{array}{l}\text { Core, section, } \\
\text { interval }(\mathrm{cm})\end{array}$ & $\begin{array}{l}\text { Top depth } \\
\text { CSF-A (m) }\end{array}$ & $\begin{array}{l}\text { Middle depth } \\
\text { CSF-A }(\mathrm{m})\end{array}$ & $\begin{array}{l}\text { Bottom depth } \\
\text { CSF-A (m) }\end{array}$ & $\begin{array}{c}\delta^{13} \mathrm{C} \\
(\% \text { VPDB) }\end{array}$ & $\begin{array}{c}\delta^{18} \mathrm{O} \\
(\% \circ \mathrm{VPDB})\end{array}$ \\
\hline $54 X-3,94-96$ & 460.15 & 460.25 & 460.35 & -1.053 & 3.379 \\
\hline $54 X-4,19-21$ & 460.90 & 461.00 & 461.10 & -0.137 & 1.962 \\
\hline $54 X-5,19-21$ & 462.40 & 462.50 & 462.60 & -0.264 & 2.460 \\
\hline $55 X-1,19-21$ & 466.00 & 466.10 & 466.20 & -0.326 & 2.551 \\
\hline $55 X-2,17-19$ & 467.48 & 467.58 & 467.68 & 0.340 & 2.305 \\
\hline $55 X-3,19-21$ & 469.00 & 469.10 & 469.20 & -0.912 & 3.217 \\
\hline $56 X-1,19-21$ & 475.60 & 475.70 & 475.80 & -0.155 & 2.825 \\
\hline $56 X-1,94-96$ & 476.35 & 476.45 & 476.55 & -0.364 & 3.195 \\
\hline $56 X-2,94-96$ & 477.85 & 477.95 & 478.05 & -0.264 & 3.240 \\
\hline $56 X-3,94-96$ & 479.35 & 479.45 & 479.55 & 0.520 & 1.455 \\
\hline $56 X-4,94-96$ & 480.85 & 480.95 & 481.05 & 0.568 & 1.621 \\
\hline $56 X-5,19-21$ & 481.60 & 481.70 & 481.80 & 0.298 & 1.319 \\
\hline $56 X-5,94-96$ & 482.35 & 482.45 & 482.55 & -0.017 & 2.057 \\
\hline $56 X-6,94-96$ & 483.85 & 483.95 & 484.05 & -0.194 & 3.256 \\
\hline $57 X-1,19-21$ & 485.20 & 485.30 & 485.40 & -0.482 & 3.198 \\
\hline $57 X-2,94-96$ & 487.45 & 487.55 & 487.65 & -0.805 & 3.140 \\
\hline $57 X-4,19-21$ & 489.70 & 489.80 & 489.90 & 0.353 & 1.338 \\
\hline $58 X-2,94-96$ & 495.77 & 495.87 & 495.97 & -0.779 & 3.117 \\
\hline $58 X-3,19-21$ & 496.52 & 496.62 & 496.72 & -1.122 & 3.269 \\
\hline $58 X-3,94-96$ & 497.27 & 497.37 & 497.47 & -1.157 & 3.337 \\
\hline $58 X-5,95-97$ & 500.28 & 500.38 & 500.48 & -1.539 & 3.194 \\
\hline $58 X-6,19-21$ & 501.02 & 501.12 & 501.22 & -1.203 & 3.143 \\
\hline $58 X-6,94-96$ & 501.77 & 501.87 & 501.97 & -0.931 & 3.306 \\
\hline $58 X-7,19-21$ & 502.52 & 502.62 & 502.72 & -1.217 & 3.274 \\
\hline $60 X-1,18-20$ & 513.99 & 514.09 & 514.19 & 0.205 & 2.312 \\
\hline $60 X-C C, 19-20$ & 514.71 & 514.81 & 514.91 & -1.952 & 3.381 \\
\hline $61 X-1,19-21$ & 523.60 & 523.70 & 523.80 & -1.067 & 3.674 \\
\hline $61 X-2,19-21$ & 524.60 & 524.70 & 524.80 & 0.127 & 2.129 \\
\hline $62 X-1,19-21$ & 533.20 & 533.30 & 533.40 & -1.248 & 3.418 \\
\hline $62 X-1,84-86$ & 533.85 & 533.95 & 534.05 & -1.937 & 3.466 \\
\hline $62 X-2,19-21$ & 534.70 & 534.80 & 534.90 & -1.757 & 3.464 \\
\hline $62 X-2,94-96$ & 535.45 & 535.55 & 535.65 & -1.392 & 3.533 \\
\hline $62 X-3,19-21$ & 536.20 & 536.30 & 536.40 & -1.294 & 3.466 \\
\hline $62 X-3,94-96$ & 536.95 & 537.05 & 537.15 & -1.648 & 3.403 \\
\hline $62 X-4,19-21$ & 537.70 & 537.80 & 537.90 & -1.130 & 3.377 \\
\hline $62 X-4,94-96$ & 538.45 & 538.55 & 538.65 & -0.898 & 3.332 \\
\hline $62 X-5,19-21$ & 539.20 & 539.30 & 539.40 & -0.821 & 3.293 \\
\hline $62 X-5,94-96$ & 539.95 & 540.05 & 540.15 & -1.064 & 3.464 \\
\hline $62 X-6,94-96$ & 541.45 & 541.55 & 541.65 & -1.308 & 3.577 \\
\hline $62 X-7,19-21$ & 541.70 & 541.80 & 541.90 & -1.744 & 3.414 \\
\hline
\end{tabular}

Depths plotted in Figures F2-F6 are the depths to middle of each sample interval. VPDB = Vienna Peedee belemnite. 
Table T3. Oxygen isotopic ratios $\left(\delta^{18} \mathrm{O}\right)$ and stable carbon isotopic ratios $\left(\delta^{13} \mathrm{C}\right)$ of Nanionella flemingi and Uvigerina perigrina (88 sample subset), Hole U1352B. (Continued on next page.)

\begin{tabular}{|c|c|c|c|c|c|c|c|}
\hline $\begin{array}{l}\text { Core, section, } \\
\text { interval }(\mathrm{cm})\end{array}$ & $\begin{array}{l}\text { Top depth } \\
\text { CSF-A (m) }\end{array}$ & $\begin{array}{l}\text { Middle depth } \\
\text { CSF-A (m) }\end{array}$ & $\begin{array}{l}\text { Bottom depth } \\
\text { CSF-A (m) }\end{array}$ & $\begin{array}{c}\delta^{18} \mathrm{O} \\
(\mathrm{N} . \text { flemingi) } \\
(\% \circ \mathrm{VPDB})\end{array}$ & $\begin{array}{c}\delta^{13} \mathrm{C} \\
\text { (N. flemingi) } \\
\text { (\%o VPDB) }\end{array}$ & $\begin{array}{c}\delta^{18} \mathrm{O} \\
(U . \text { peregrina }) \\
\text { (\%o VPDB) }\end{array}$ & $\begin{array}{c}\delta^{13} \mathrm{C} \\
\text { (U. peregrine) } \\
\text { (\%o VPDB) }\end{array}$ \\
\hline \multicolumn{8}{|l|}{ 317-U1352B- } \\
\hline $18 \mathrm{H}-1,19-21$ & 156.39 & 156.40 & 156.41 & 1.838 & -0.460 & 2.162 & 0.380 \\
\hline $18 \mathrm{H}-3,19-21$ & 159.39 & 159.40 & 159.41 & 3.738 & -0.002 & 3.562 & 0.564 \\
\hline $20 \mathrm{H}-3,19-21$ & 177.57 & 177.58 & 177.59 & 3.573 & -0.237 & 3.117 & 0.195 \\
\hline $21 \mathrm{H}-5,94-96$ & 186.64 & 186.65 & 186.66 & 3.252 & -0.097 & 3.004 & 0.583 \\
\hline $21 \mathrm{H}-6,94-96$ & 188.14 & 188.15 & 188.16 & 3.542 & 0.001 & 2.969 & 0.082 \\
\hline $22 \mathrm{H}-2,19-21$ & 190.89 & 190.90 & 190.91 & 3.189 & 0.101 & 3.027 & 0.357 \\
\hline $22 \mathrm{H}-6,19-21$ & 196.89 & 196.90 & 196.91 & 3.270 & -0.085 & 3.066 & 0.311 \\
\hline $22 \mathrm{H}-7,19-21$ & 198.39 & 198.40 & 198.41 & 3.076 & 0.292 & 2.591 & 0.287 \\
\hline $23 \mathrm{H}-1,94-96$ & 199.64 & 199.65 & 199.66 & 3.145 & 0.243 & 2.818 & 0.304 \\
\hline $23 \mathrm{H}-2,19-21$ & 200.39 & 200.40 & 200.41 & 3.235 & 0.099 & 2.870 & 0.510 \\
\hline $23 \mathrm{H}-2,94-96$ & 201.14 & 201.15 & 201.16 & 3.916 & -0.468 & 3.406 & -0.181 \\
\hline $23 \mathrm{H}-6,19-21$ & 206.39 & 206.40 & 206.41 & 3.489 & -0.061 & 2.657 & 0.070 \\
\hline $23 \mathrm{H}-6,94-96$ & 207.14 & 207.15 & 207.16 & 3.295 & -0.107 & 2.755 & 0.406 \\
\hline $23 \mathrm{H}-7,19-21$ & 207.89 & 207.90 & 207.91 & 3.281 & 0.289 & 3.060 & 0.444 \\
\hline $24 \mathrm{H}-4,19-21$ & 212.89 & 212.90 & 212.91 & 3.506 & -0.106 & 3.198 & 0.360 \\
\hline $27 \mathrm{H}-1,19-21$ & 236.89 & 236.90 & 236.91 & 2.955 & 0.102 & 3.177 & 0.547 \\
\hline $27 \mathrm{H}-5,94-96$ & 243.64 & 243.65 & 243.66 & 3.118 & 0.004 & 2.610 & 0.276 \\
\hline $27 \mathrm{H}-6,64-66$ & 244.84 & 244.85 & 244.86 & 2.696 & -0.148 & 2.662 & 0.335 \\
\hline $27 \mathrm{H}-7,19-21$ & 245.39 & 245.40 & 245.41 & 2.619 & -0.013 & 2.698 & 0.262 \\
\hline $28 \mathrm{H}-1,19-21$ & 246.39 & 246.40 & 246.41 & 3.274 & -0.201 & 2.400 & 0.135 \\
\hline $28 \mathrm{H}-2,94-96$ & 248.57 & 248.58 & 248.59 & 3.603 & 0.065 & 3.270 & 0.002 \\
\hline $28 \mathrm{H}-3,19-21$ & 249.32 & 249.33 & 249.34 & 3.070 & -0.306 & 2.682 & -0.336 \\
\hline $28 \mathrm{H}-3,34-36$ & 249.47 & 249.48 & 249.49 & 2.627 & -0.167 & 2.758 & 0.027 \\
\hline $28 \mathrm{H}-3,94-96$ & 250.07 & 250.08 & 250.09 & 3.011 & -0.390 & 2.900 & -0.191 \\
\hline $29 \mathrm{H}-2,19-21$ & 252.89 & 252.90 & 252.91 & 3.544 & -1.025 & 3.177 & -0.159 \\
\hline $32 \mathrm{H}-5,19-21$ & 278.34 & 278.35 & 278.36 & 3.061 & -0.089 & 2.734 & -0.064 \\
\hline $33 \mathrm{H}-1,19-21$ & 281.89 & 281.90 & 281.91 & 2.897 & 0.083 & 2.566 & 0.038 \\
\hline $33 \mathrm{H}-1,93-95$ & 282.63 & 282.64 & 282.65 & 3.254 & -0.233 & 3.231 & 0.028 \\
\hline $33 \mathrm{H}-2,19-21$ & 283.3 & 283.31 & 283.32 & 3.481 & -0.172 & 2.792 & -0.099 \\
\hline $33 \mathrm{H}-3,19-21$ & 284.07 & 284.08 & 284.09 & 3.297 & -0.248 & 2.692 & 0.154 \\
\hline $33 \mathrm{H}-4,94-96$ & 285.58 & 285.59 & 285.60 & 3.287 & -0.416 & 3.308 & 0.053 \\
\hline $33 \mathrm{H}-5,19-21$ & 286.33 & 286.34 & 286.35 & 3.371 & -0.407 & 3.068 & -0.228 \\
\hline $34 \mathrm{H}-4,23-25$ & 291.00 & 291.01 & 291.02 & 3.188 & -0.450 & 2.057 & 0.260 \\
\hline $35 \mathrm{H}-1,36-38$ & 293.26 & 293.27 & 293.28 & 3.034 & -0.130 & 3.087 & 0.327 \\
\hline $35 \mathrm{H}-2,19-21$ & 294.53 & 294.54 & 294.55 & 2.710 & 0.140 & 2.297 & 0.225 \\
\hline $36 \mathrm{H}-2,19-21$ & 296.69 & 296.70 & 296.71 & 3.137 & 0.296 & 2.652 & 0.282 \\
\hline $37 X-1,94-96$ & 297.94 & 297.95 & 297.96 & 2.035 & 0.767 & 2.152 & 0.781 \\
\hline $37 X-2,19-21$ & 298.69 & 298.70 & 298.71 & 3.043 & 0.035 & 2.989 & -0.131 \\
\hline $38 X-2,94-96$ & 305.04 & 305.05 & 305.06 & 3.223 & -0.212 & 2.675 & 0.346 \\
\hline $39 X-7,19-21$ & 321.19 & 321.20 & 321.21 & 3.235 & 0.323 & 3.178 & 0.584 \\
\hline $40 X-6,94-96$ & 330.34 & 330.35 & 330.36 & 2.607 & 0.161 & 2.223 & 0.460 \\
\hline $41 X-1,19-21$ & 331.69 & 331.70 & 331.71 & 3.258 & -0.207 & 2.255 & 0.385 \\
\hline $41 X-2,94-96$ & 333.94 & 333.95 & 333.96 & 3.595 & -0.600 & 2.770 & 0.399 \\
\hline $41 X-3,94-96$ & 335.44 & 335.45 & 335.46 & 3.389 & -0.141 & 3.356 & 0.115 \\
\hline $41 X-4,94-96$ & 336.94 & 336.95 & 336.96 & 3.530 & -0.278 & 2.860 & -0.087 \\
\hline $42 X-4,94-96$ & 346.54 & 346.55 & 346.56 & 2.808 & -0.203 & 2.741 & 0.199 \\
\hline $42 X-5,18-20$ & 347.28 & 347.29 & 347.30 & 1.345 & 0.837 & 1.548 & 0.828 \\
\hline $42 X-5,94-96$ & 348.04 & 348.05 & 348.06 & 3.008 & 0.092 & 2.708 & -0.337 \\
\hline $43 X-1,20-22$ & 350.90 & 350.91 & 350.92 & 3.950 & -0.962 & 3.896 & -0.441 \\
\hline $43 X-1,95-97$ & 351.65 & 351.66 & 351.67 & 4.028 & -0.836 & 3.998 & -0.361 \\
\hline $43 X-2,19-21$ & 352.39 & 352.40 & 352.41 & 3.828 & -1.254 & 3.419 & -1.222 \\
\hline $43 X-4,19-21$ & 355.39 & 355.40 & 355.41 & 2.753 & 0.021 & 2.579 & 0.557 \\
\hline $43 X-6,94-96$ & 359.14 & 359.15 & 359.16 & 3.282 & 0.092 & 2.968 & 0.276 \\
\hline $44 X-6,94-96$ & 368.84 & 368.85 & 368.86 & 2.793 & 0.340 & 3.009 & 0.739 \\
\hline $45 X-3,94-96$ & 373.94 & 373.95 & 373.96 & 3.134 & 0.002 & 2.884 & 0.209 \\
\hline $45 X-4,94-96$ & 375.44 & 375.45 & 375.46 & 2.899 & -0.100 & 2.850 & 0.253 \\
\hline $45 X-5,19-21$ & 376.19 & 376.20 & 376.21 & 3.628 & 0.045 & 3.190 & 0.754 \\
\hline $46 X-3,19-21$ & 382.77 & 382.78 & 382.79 & 3.046 & -0.243 & 2.978 & 0.233 \\
\hline $47 X-1,18-20$ & 389.38 & 389.39 & 389.40 & 3.183 & -0.132 & 2.362 & 0.178 \\
\hline $47 X-2,19-21$ & 390.89 & 390.90 & 390.91 & 2.967 & -0.108 & 2.309 & 0.226 \\
\hline $47 X-2,93-95$ & 391.63 & 391.64 & 391.65 & 2.217 & 0.349 & 1.786 & 0.337 \\
\hline $47 X-3,19-21$ & 392.39 & 392.40 & 392.41 & 3.018 & -0.269 & 2.198 & -0.072 \\
\hline $47 X-6,18-20$ & 396.88 & 396.89 & 396.90 & 2.883 & -0.591 & 2.981 & 0.091 \\
\hline $48 X-3,94-96$ & 402.64 & 402.65 & 402.66 & 2.993 & -0.351 & 2.697 & 0.511 \\
\hline $48 X-4,19-21$ & 403.39 & 403.40 & 403.41 & 2.806 & -0.161 & 2.497 & 0.234 \\
\hline
\end{tabular}


Table T3 (continued).

\begin{tabular}{|c|c|c|c|c|c|c|c|}
\hline $\begin{array}{l}\text { Core, section, } \\
\text { interval }(\mathrm{cm})\end{array}$ & $\begin{array}{l}\text { Top depth } \\
\text { CSF-A (m) }\end{array}$ & $\begin{array}{l}\text { Middle depth } \\
\text { CSF-A (m) }\end{array}$ & $\begin{array}{l}\text { Bottom depth } \\
\text { CSF-A (m) }\end{array}$ & $\begin{array}{c}\delta^{18} \mathrm{O} \\
(\mathrm{N} . \text { flemingi) } \\
(\% \text { o VPDB) }\end{array}$ & $\begin{array}{c}\delta^{13} \mathrm{C} \\
(\mathrm{N} . \text { flemingi) } \\
(\% \text { VPDB) }\end{array}$ & $\begin{array}{c}\delta^{18} \mathrm{O} \\
(U . \text { peregrina }) \\
(\% \text { VPDB })\end{array}$ & $\begin{array}{c}\delta^{13} \mathrm{C} \\
\text { (U. peregrine) } \\
\text { (\%o VPDB) }\end{array}$ \\
\hline $49 X-3,93-95$ & 412.23 & 412.24 & 412.25 & 2.224 & -0.214 & 2.586 & 0.326 \\
\hline $50 X-4,19-21$ & 422.59 & 422.60 & 422.61 & 1.682 & 0.520 & 2.182 & 0.216 \\
\hline $50 X-7,19-21$ & 426.89 & 426.90 & 426.91 & 2.925 & 0.527 & 2.475 & 0.435 \\
\hline $51 X-3,95-97$ & 431.45 & 431.46 & 431.47 & 3.924 & -0.400 & 3.367 & -0.385 \\
\hline $52 X-3,19-21$ & 440.29 & 440.30 & 440.31 & 3.397 & -0.296 & 2.872 & 0.200 \\
\hline $52 X-5,93-95$ & 444.03 & 444.04 & 444.05 & 1.882 & 0.167 & 1.869 & 0.311 \\
\hline $52 X-6,94-96$ & 445.54 & 445.55 & 445.56 & 2.647 & -0.213 & 2.622 & 0.200 \\
\hline $53 X-1,19-21$ & 446.89 & 446.90 & 446.91 & 2.749 & -0.001 & 2.322 & 0.024 \\
\hline $53 X-1,95-97$ & 447.65 & 447.66 & 447.67 & 2.004 & 0.442 & 1.643 & 0.527 \\
\hline $53 X-2,94-96$ & 449.14 & 449.15 & 449.16 & 1.953 & 0.071 & 1.649 & 0.280 \\
\hline $53 X-3,94-96$ & 450.64 & 450.65 & 450.66 & 1.388 & 0.760 & 1.492 & 0.288 \\
\hline $53 X-4,19-21$ & 451.39 & 451.40 & 451.41 & 2.605 & -0.115 & 1.820 & 0.168 \\
\hline $53 X-5,19-21$ & 452.89 & 452.90 & 452.91 & 2.641 & -0.160 & 1.910 & -0.009 \\
\hline $53 X-5,94-96$ & 453.64 & 453.65 & 453.66 & 3.383 & -0.262 & 2.588 & -0.357 \\
\hline $53 X-6,95-97$ & 455.12 & 455.13 & 455.14 & 3.474 & -0.848 & 3.291 & -0.763 \\
\hline $54 X-2,94-96$ & 458.74 & 458.75 & 458.76 & 2.588 & -0.437 & 2.683 & -0.150 \\
\hline $54 X-3,94-96$ & 460.24 & 460.25 & 460.26 & 3.379 & -1.053 & 3.354 & -0.892 \\
\hline $54 X-5,19-21$ & 462.49 & 462.50 & 462.51 & 2.460 & -0.264 & 2.360 & -0.057 \\
\hline $56 X-3,94-96$ & 479.44 & 479.45 & 479.46 & 1.455 & 0.520 & 1.272 & 0.365 \\
\hline $56 X-5,19-21$ & 481.69 & 481.70 & 481.71 & 1.319 & 0.298 & 1.718 & 0.407 \\
\hline $58 X-3,19-21$ & 496.61 & 496.62 & 496.63 & 3.269 & -1.122 & 3.362 & -0.152 \\
\hline $58 X-6,19-21$ & 501.11 & 501.12 & 501.13 & 3.143 & -1.203 & 2.596 & -0.749 \\
\hline
\end{tabular}

VPDB $=$ Vienna Peedee belemnite.

Table T4. Bioevents reexamined in this study (HO Pseudoemiliania lacunosa) and reported during Expedition 317 (all others; see the "Methods" chapter [Expedition 317 Scientists, 2011a]).

\begin{tabular}{|c|c|c|c|c|c|c|}
\hline \multirow[b]{2}{*}{ Age (Ma) } & \multirow[b]{2}{*}{ Bioevent } & \multirow[b]{2}{*}{ Age reference } & \multicolumn{2}{|c|}{ Depth CSF-A (m) } & \multicolumn{2}{|c|}{ Core, section } \\
\hline & & & Top & Bottom & Top & Bottom \\
\hline & & & & & 317-U1352B & 317-U1352B- \\
\hline 0.29 & LO Emiliania huxleyi (NN21 base) & Lourens et al. (2004) & 112.82 & 121.10 & $12 \mathrm{H}-\mathrm{CC}$ & $13 \mathrm{H}-\mathrm{CC}$ \\
\hline 0.44 & HO Pseudoemiliania lacunosa (NN20 base) & Lourens et al. (2004) & 129.77 & 130.34 & $14 \mathrm{H}-6$ & $14 \mathrm{H}-\mathrm{CC}$ \\
\hline 0.91 & HCO Reticulofenestra asanoi & Lourens et al. (2004) & 257.09 & 266.92 & $29 \mathrm{H}-\mathrm{CC}$ & $30 \mathrm{H}-\mathrm{CC}$ \\
\hline 1.24 & HO Gephyrocapsa >6.5 $\mu \mathrm{m}$ & de Kaenel et al. (1999) & 348.59 & 360.08 & $42 \mathrm{X}-\mathrm{CC}$ & $43 \mathrm{X}-\mathrm{CC}$ \\
\hline 1.26 & HO Gephyrocapsa $>5.5 \mu \mathrm{m}$ & Lourens et al. (2004) & 360.08 & 369.96 & $43 \mathrm{X}-\mathrm{CC}$ & $44 \mathrm{X}-\mathrm{CC}$ \\
\hline 1.56 & LO Gephyrocapsa >5.5 $\mu \mathrm{m}$ & Lourens et al. (2004) & 412.30 & 427.34 & $49 \mathrm{X}-\mathrm{CC}$ & $50 \mathrm{X}-\mathrm{CC}$ \\
\hline 1.69 & HO Gephyrocapsa >4 $\mu \mathrm{m}$ & Lourens et al. (2004) & 463.67 & 469.84 & $54 \mathrm{X}-\mathrm{CC}$ & $55 \mathrm{X}-\mathrm{CC}$ \\
\hline \multirow[t]{2}{*}{1.73} & LO Gephyrocapsa caribbeanica & de Kaenel et al. (1999) & 469.84 & 484.83 & $55 \mathrm{X}-\mathrm{CC}$ & $56 \mathrm{X}-\mathrm{CC}$ \\
\hline & Hiatus & & 491.74 & 525.34 & $57 \mathrm{X}-\mathrm{CC}$ & $61 X-C C$ \\
\hline 2.78 & HO Reticulofenestra ampla & Kameo and Bralower (2000) & 525.34 & 542.58 & $61 X-C C$ & $62 \mathrm{X}-\mathrm{CC}$ \\
\hline
\end{tabular}

$\mathrm{LO}=$ lowest occurrence, $\mathrm{HO}=$ highest occurrence, $\mathrm{HCO}=$ highest common occurrence . 